\title{
Spatial effects and strategic behaviour in a multiregional transboundary pollution dynamic game*
}

\author{
Javier de Frutos G Guiomar Martín-Herrán
}

IMUVA, Universidad de Valladolid. Spain.

\begin{abstract}
We analyze a transboundary pollution differential game where pollution control is spatially distributed among a number of agents with predetermined spatial relationships. The analysis emphasizes, first, the effects of the different geographical relationships among decision makers; and second, the strategic behaviour of the agents. The dynamic game considers a pollution stock (the state variable) distributed among one large region divided in subregions which control their own emissions of pollutants. The emissions are also represented as distributed variables. The dynamics of the pollution stock is defined by a parabolic partial differential equation. We numerically characterize the feedback Nash equilibrium of a discrete-space model that still captures the spatial interactions among agents. We evaluate the impact of the strategic and spatially dynamic behaviour of the agents on the design of equilibrium environmental policies.
\end{abstract}

Keywords: Transboundary Pollution; Spatial Dynamics; Spatially Distributed Controls; Differential Games; Parabolic Differential Equations.

JEL Codes: Q5; R12; C73; C61.

*The authors would like to thank H. Benchekroun and participants at SURED 2016 (Banyuls sur Mer), EAERE 2016 (Zurich), GeComplexity Conference 2016 (Heraklion) and the 17th International Symposium on Dynamic Games and Applications (Urbino) for stimulating discussions. This research is partially supported by MEC under projects MTM2013-42538-P (first author) and ECO2014-52343-P (second author), co-financed by FEDER funds. The authors thank the support of European Cooperation in Science and Technology through COST Action IS1104, "The EU in the new complex geography of economic systems: models, tools and policy evaluation". 


\section{Introduction}

The standard dynamic models used in the literature to study different types of economic and environmental problems have restricted their attention to time. These models ignore space even when studying problems with an important geographic flavour. However, it seems natural to try to extend the analysis to a spatial dimension in order to describe in a more realistic manner the world's economic and environmental problems. The technical difficulties that arise when optimization takes place in spatio-temporal domains are undoubtedly the reason for the lack of abundant literature on this subject. Recently some authors have introduced in different economic contexts (for example, allocation of economic activity; technological diffusion; environmental and climate problems) this spatial dimension. These authors have analyzed finite or infinite time optimal control problems extended to infinite dimensional state space. These contributions have focussed either on the problem of a social planner or on special private optimization problems. In the first case, the social planner allocates resources in order to maximize the present value of an objective over the entire spatial domain subject to the spatio-temporal evolution of the state variable. In the second case, particular assumptions either on the economic agents' behaviour or on the diffusion process are made. These assumptions imply that the agents behave myopically in both the temporal and the spatial dimensions, in the sense that they do not care to take the future allocation paths into account. As a result agents solve static problems. To the best of our knowledge there are no previous studies that consider agents who behave both dynamically and strategically. This paper fills this gap in the literature and presents a first approach to characterize the equilibrium outcomes of an intertemporal transboundary pollution dynamic game where there is a continuum of spatial sites and the pollution stock diffuses over these sites.

The previous contributions in this area can be classified in two broad groups. First, the works focussing on economic growth theory with spatial diffusion; and second, the contributions which explore the spatial dimension in environmental and resource economics. In the first group the diffusion mechanisms involved are production factor mobility or technological diffusion, while in the second group are diffusion of a pollutant or the distribution of the biomass of a natural resource. The following is a no exhaustive list of works which can be classified in the first group: Brito (2004), Boucekkine et al. (2009, 2013a, 2013b), Camacho et al. (2008), Brock \& Xepapadeas (2008a), Brock et al. (2014a) and Fabbri (2016). A list of papers belonging to the second group of the literature includes: Brock \& Xepapadeas (2008b, 2010), Brock et al. (2014b), Camacho \& Pérez-Barahona (2015), Xepapadeas (2010). Finally, there are some recent studies (among others, Anita et al. (2013), Yamaguchi (2014), Desmet \& Rossi-Hansberg (2015), and La Torre et al. (2015)) which could be classified in both groups. These works have put the focus on the analysis of problems coupling capital accumulation and pollution 
diffusion.

Brito (2004), Boucekkine et al. (2009, 2013a), Camacho et al. (2008) and Fabbri (2016) analyze optimal dynamic social welfare for spatial economic growth models. In all cases a policy maker maximizes consumers' well-being, capital is mobile across space and household's budget is governed by a parabolic partial differential equation (PDE). Brock \& Xepapadeas (2008a) also study the optimal spatial allocation of economic activity in a dynamic setting with capital accumulation. Unlike the aforementioned papers there is no capital mobility and the spatial component is introduced through technological diffusion. All these papers use extended versions of Pontryagin's maximum principle to obtain necessary conditions for the optimization problems at hand and usually focus on the problem of a social planner who allocates resources. Exceptions are Brock \& Xepapadeas (2008a) and Brock et al. (2014a) who also study the problem where an economic agent considers certain external effects as outside his control and treats them as exogenous. Therefore, there is an incomplete internalization of the spatial externality by the optimizing agents. Desmet \& Rossi-Hansberg (2010) surveyed all this research devoted to the analysis of spatial economic growth models. Boucekkine et al. (2013b) present a survey of the use of parabolic PDEs in economic growth theory.

One of the first contributions to the stream of the literature that explores the spatial dimension in environmental and resource economics is Brock \& Xepapadeas (2008b). The results on local stability analysis for infinite horizon optimal control problems adapted to their spatial context are illustrated with two applications. First, optimal ecosystem management model, where the ecosystems are spatially connected; and second, renewable resource harvesting models, where the resource itself diffuses across space. Brock \& Xepapadeas (2010) apply their results on pattern formation to the management and regulation of a semi-arid system assuming two different settings. First, economic agents maximize myopic profits and ignore spillovers onto agents at other sites. Second, a social planner internalizes these spillovers. As the authors already noted due to the myopic assumptions regarding agents' behaviour, the private optimum discussed in their paper represents a series of static optimization problems defined at each point in time and space. The same assumptions on myopic agents' behaviour are considered in Xepapadeas (2010). This paper revises the tools for studying the interactions of pattern formation and agglomeration mechanisms in optimal control problems with applications to resource management. Brock et al. (2014b) revisit these methods and tools in their review of the applications of optimal control of diffusive transport processes to environmental and climate problems in economics. Camacho \& Pérez-Barahona (2015) analyze optimal land use from a social planner's point of view. The social planner decides the land use activities taking into account that local actions affect the whole space because pollution flows across locations resulting on both local and global damages. 
Among the different examples presented in Brock et al. (2014b), the example closest to the problem which concerns us corresponds to the pollution control in a spatial setting. The PDE describing the evolution and diffusion of pollutants in the environment in Brock et al. (2014b) is similar to the specification of the PDE describing this evolution in our problem. However, we have a two-dimensional equation (theirs is one dimensional) which is an important feature when the geographical or spatial aspect of the problem is taken into account. Apart from this difference in spatio-temporal variability of the stock of pollutant, the main difference comes from different settings concerning the optimizing agents. Brock et al. (2014b) consider an environmental regulator who seeks to maximize the discounted benefits net of environmental damages due to the concentration of pollutants over the entire spatial domain subject to the spatio-temporal evolution of the stock of a pollutant. Our paper studies dynamic optimization for the pollution control in a spatial setting with strategic agents and focuses on the equilibrium emission strategies in a multiregional setting. An essential difference with respect to the previous literature is that we consider that each economic agent responsible for controlling the emissions at each region takes into account the spatial transport phenomena across space in order to maximize his profits.

Our contribution to the literature is twofold. First, we add the spatial aspect to the literature on transboundary pollution dynamic games (Ploeg and Zeeuw (1992) and Dockner and Long (1993) are seminal papers in this area, and Jørgensen et al. (2010) surveyed this literature). Second, we add the strategic aspect to the literature on spatial economics, and in particular, to the pollution control in a spatial setting described in the previous paragraph.

Building on these two branches of the literature, the objective of this paper is to investigate the impact of the strategic and spatially dynamic behaviour of the economic agents responsible for controlling the emissions of pollutant on the design of equilibrium environmental policies. More specifically, we aim at answering the following research questions:

1. In a transboundary pollution dynamic game setting, do the environmental policies that take into account the spatial context differ from those that ignore the spatial transport phenomena?

2. Considering the pollution control in a spatial setting, do the environmental policies that take into account the strategic behaviour of the decision agents differ from those fixed by an environmental regulator?

In order to answer our research questions we state and analyze a problem for multiregional spatially distributed control of pollution. We state the model in continuous space and continuous time with two spatial dimensions and one time dimension. The planar region of interest is divided in $J$ subregions. In 
each subregion there is a decision-maker who decides the emission level. His objective is to maximize the present value of benefits net of environmental damages due to the concentration of pollutants over the spatial subdomain corresponding to his subregion. His optimization problem is subject to the spatiotemporal evolution of the stock of a pollutant. This spatio-temporal evolution is described by a Diffusion PDE and general boundary conditions are assumed. Summarizing, our specification corresponds to a $J$ player differential game with one control variable for each player (the emission decision at his subregion) and one infinite-dimensional state variable (the stock of a pollutant). Each player decides his net profits maximizing level of emission at each spatial point located in his subregion and at each time. In his maximization problem takes into account the PDE describing the spatio-temporal evolution of the stock of a pollutant. It is worth noting that the literature on spatial economics previously discussed seeking private equilibria, the economic agents act spatially myopically by ignoring spatial transport. In our formulation each player does not ignore the spatial aspect in his optimization problem. We characterize Markov-perfect Nash equilibria of the differential game.

As a first approach to characterize the equilibrium outcomes of the transboundary pollution dynamic game with spatial effects, and in order to overcome the difficulties arising from the infinite dimensionality of the model we consider a simplified model capturing the spatial interactions. This simplified model can be seen as a spatial discretization of the original model. This space-discretized model has $J$ players and $J$ state variables, each one describing the average pollution in one subregion. Each player has one control variable which is the average total emission in his subregion. Furthermore, when making profit maximizing emission decision at this subregion takes into account the time evolution of the $J$ state variables. The time dynamics of the state variables is described by a system of $J$ ordinary differential equations (ODEs). This space-discretized formulation fits the structure used by Mäler \& Zeeuw (1998) in their analysis of the acid rain differential game. Recently, Graß \& Uecker (2017) apply a similar spatial discretization approach to transform a system of PDEs into a very large system of ODEs. They numerically treat spatially distributed optimal control problems with an infinite time horizon. As an example they analyze a shallow lake model with diffusion.

We analytically characterize the feedback Nash equilibrium of the two-player version of the spacediscretized model. This model is solved using a numerical algorithm adapted from De Frutos \& MartínHerrán (2015) when more than two players are considered. Essentially, the numerical algorithm solves an approximate time-discrete dynamic game. Numerical experiments are presented to illustrate the results. A first important conclusion which can be derived from our results is that in a spatial context the environmental policies might be very different from the traditional policies which ignore either the spatial transport phenomena or the strategic behaviour of the decision makers. 
The paper is organized as follows. In the next section we present the multiregional spatially distributed control of pollution formulated initially as a continuous-space model, and in a second step, as a discrete-space model. Section 3 presents some numerical examples highlighting some properties of the Markov-perfect Nash equilibria of the model and their main differences with the formulation either without spatial effects or without strategic interactions among the players. Section 4 is devoted to present some concluding remarks. The characterization of the Markov-perfect Nash equilibrium of the model, the derivation of the discrete-space model as well as the description of the numerical method are relegated to the Appendix.

\section{The model}

The model is a $J$-player non-cooperative differential game. Let us consider a planar region $\Omega$ with a given partition in $J$ subdomains (subregions) $\Omega_{j}, j=1, \ldots, J$, satisfying

$$
\bar{\Omega}=\bigcup_{j=1}^{J} \bar{\Omega}_{j}, \quad \Omega_{i} \cap \Omega_{j}=\emptyset, \quad i \neq j,
$$

with $\bar{\Omega}$ the closure of $\Omega$. Let us denote $\partial_{i j}$ the common boundary between subdomains $\Omega_{i}$ and $\Omega_{j}$,

$$
\partial_{i j}:=\partial \Omega_{i} \cap \partial \Omega_{j}=\bar{\Omega}_{i} \cap \bar{\Omega}_{j}, \quad i \neq j .
$$

Player (subregion) $i$ wishes to choose the rate of pollutant emissions in subregion $\Omega_{i}$ to maximize his own payoff. Let us denote by $u_{i}(\boldsymbol{x}, t), i=1, \ldots, J$, the emission rate of subregion $i$ at time $t \geq 0$ at the particular point of the region $\boldsymbol{x} \in \Omega$. It is convenient to think of $u_{i}(\boldsymbol{x}, t)$ as a density of emission rates which are distributed along the subregion $\Omega_{i}$. We denote by $P(\boldsymbol{x}, t)$ the stock of pollution defined for all $\boldsymbol{x} \in \Omega$. Along the paper the symbol $\nabla f$ denotes the spatial gradient of a scalar function $f: \Omega \rightarrow \mathbb{R}$,

and the symbol $\nabla \cdot \boldsymbol{f}=\frac{\partial f_{1}}{\partial x}+\frac{\partial f_{2}}{\partial y}$ represents the divergence of a vectorial function $\boldsymbol{f}=\left[f_{1}, f_{2}\right]: \Omega \rightarrow \mathbb{R}^{2}$.

The spatio-temporal dynamics of the stock of pollution is given by the following parabolic partial differential equation:

$$
\begin{aligned}
& \frac{\partial P}{\partial t}=\nabla \cdot(k \nabla P)-c P+F(\boldsymbol{u}), \boldsymbol{x} \in \Omega, \\
& P(\boldsymbol{x}, 0)=P_{0}(\boldsymbol{x}), \quad \boldsymbol{x} \in \Omega, \\
& \alpha(\boldsymbol{x}) P(\boldsymbol{x}, t)+k(\boldsymbol{x}) \nabla P^{T}(\boldsymbol{x}, t) \boldsymbol{n}=\alpha(\boldsymbol{x}) P_{b}(\boldsymbol{x}, t), \quad \boldsymbol{x} \in \partial \Omega .
\end{aligned}
$$

Here $\boldsymbol{u}=\left[u_{1}, \ldots, u_{J}\right]^{T}$ is the vector of emission rates, $k=k(\boldsymbol{x})$ is a local diffusion coefficient which is assumed to be a smooth function satisfying $k_{m} \leq k(\boldsymbol{x}) \leq k_{M}$, for all $\boldsymbol{x} \in \Omega$, where $0<k_{m} \leq k_{M}$ are two given constants. The coefficient $k=k(\boldsymbol{x})$ is a characteristic diffusion parameter that can depend on 
$\boldsymbol{x} \in \Omega$. It measures the velocity at which the stock of pollution is diffused away in a particular location $\boldsymbol{x}$. The term $c P, c=c(\boldsymbol{x}, t) \geq 0$, is a natural decay of the pollutant.

Diffusion models as that described in (3) have been used extensively in the literature (see for example, Stockie (2011)) as a mathematical description of contaminant transport in a variety of applications. Among others, atmospheric dispersion of pollutants, transport of pollutants in aquifers, groundwater pollution, or porous soils. Pollution models usually incorporate a combination of diffusion and advection effects. In a diffusive phenomenon particles flow from points with higher concentration to points with lower concentration (Fick's law). When advection is important besides the diffusive effect particles are transported due to some external convective field (as wind, water flow, etc.). In some cases a non-linear term is added to the equation. For the ease of presentation in this paper we focus on diffusion as in Brock \& Xepapadeas (2008a, 2010) and Camacho \& Pérez-Barahona (2015). See the appendices for the complete model.

We assume that only agent $j$ is emitting in subregion $\Omega_{j}, j=1, \ldots, J$. That is, there is just one agent emitting in each subregion $\Omega_{j}$. Furthermore, each $\boldsymbol{x} \in \Omega$ belongs to one and only one $\Omega_{j}$. With this hypothesis the source term $F(\boldsymbol{u})$ can be written in the form

$$
F(\boldsymbol{u}(\boldsymbol{x}, t))=\sum_{j=1}^{J} F_{j}\left(u_{j}(\boldsymbol{x}, t)\right) \mathbf{1}_{\Omega_{j}}(\boldsymbol{x}),
$$

for a given family of smooth functions $F_{j}, j=1, \ldots, J$, where $\mathbf{1}_{\Omega_{j}}$ denotes the characteristic function of set $\Omega_{j}$, that is, the function defined to be identically one on $\Omega_{j}$, and zero elsewhere. In other words $F(\boldsymbol{u}(\boldsymbol{x}, t))=F_{j}\left(u_{j}(\boldsymbol{x}, t)\right)$ for $\boldsymbol{x} \in \Omega_{j}$.

The rationale of the model is that although with the modelization assumption (4) the emission rates of subregion $j$ contribute to enlarge the stock of pollution only in subregion $j$, the diffusion process modelized by the state equation (3) transfers part of the pollution to the whole region $\Omega$. We remark that, due to the diffusive character of the state equation, the emissions in subregion $\Omega_{j}$ instantaneously affect each one of the subregions $\Omega_{i}, j \neq i$. How much the emissions of subregion $j$ do affect subregion $i$ depends on the time elapsed from the instant when the emissions take place and the distance between subregions $j$ and $i$. The second equation in (3) is the initial distribution of the stock of pollution along region $\Omega$. The last ingredient of the model is the boundary condition stated in the third equation of (3). Function $\alpha(\boldsymbol{x})$ is a non-negative smooth function that appears after applying Newton's law of diffusion on the boundary of $\Omega$. The third equation on (3) simply states that the flux of pollution throughout $\partial \Omega$ is proportional to the difference $P_{b}(\boldsymbol{x})-P(\boldsymbol{x})$, where $P_{b}(\boldsymbol{x})$ is a given function representing the concentration of pollution in the exterior of $\Omega$ and $\boldsymbol{n}$ denotes the normal vector exterior to $\Omega$. 
The objective of player $i, i=1, \ldots, J$, is to maximize his payoff

$$
J_{i}\left(u_{1}, \ldots, u_{J}, P_{0}\right)=\int_{0}^{+\infty} \int_{\Omega_{i}} e^{-\rho t} G_{i}\left(u_{1}, \ldots, u_{J}, P\right) \mathrm{d} \boldsymbol{x} \mathrm{d} t
$$

subject to the dynamics given in equation (3). Here $\rho>0$ is a given and common time-discount rate. As it is standard in dynamic pollution games (see Jørgensen et al. (2010) for a survey of this literature), the instantaneous welfare of each subregion is given by a benefit from consumption minus the damage caused by the stock of pollution. Each subregion produces a single consumption good, the production of which generates emissions. The preferences of consumers and the emission-consumption trade-off functions are such that the instantaneous benefits of the subregion is given by a function of the emission rates $B_{i}\left(u_{i}\right)$. Furthermore, the environmental damage caused by the accumulated stock of pollution is represented by function $D_{i}(P)$. Therefore, the net benefits from consumption have the form

$$
G_{i}\left(u_{1} \ldots, u_{J}, P\right)=\left(B_{i}\left(u_{i}\right)-D_{i}(P)\right) \mathbf{1}_{\Omega_{i}}
$$

for given smooth functions $B_{i}$ and $D_{i}$. As common in the literature it is assumed that $B_{i}$ and $D_{i}$ are concave and convex functions of their arguments, respectively. We remark that the payoff (5) can be seen as an average over $\Omega_{i}$ of a density of revenue represented by the function $\left(B_{i}\left(u_{i}\right)-D_{i}(P)\right) \mathbf{1}_{\Omega_{i}}$.

Assuming that the state variable can be observed and used for conditioning behaviour, we focus on stationary Markov-perfect Nash equilibria. Thus at any point in time and space the emission decision of an agent depends only on the state of the pollution stock at that moment and point in space. These stationary Markovian strategies do not require precommitment to a course of action over time and have been assumed to be a good description of realistic behaviour (see, for example, Haurie et al. (2012) and Jørgensen et al. (2010)). In Appendix A we collect the set of technical hypotheses, assumed from now on, needed to characterize the stationary Markov-perfect Nash equilibrium of the dynamic game defined by (3)-(6).

To concentrate our attention on our main research questions and focus us on the spatial relationships with respect to other decision makers we add two reasonable hypotheses. First, each decision maker is indifferent about where to produce, and hence, where to emit. In other words, the decision maker $j$ has not preference about the particular point $\boldsymbol{x} \in \Omega_{j}$ in which to emit. The technology used to produce, and consequently the profits derived from production are identical at each point in subregion $\boldsymbol{x} \in \Omega_{j}$. The second hypothesis assumes that each decision maker is indifferent to the environmental damage, in the sense that each decision maker $j$ identically values the environmental damage throughout the whole set $\Omega_{j}$. These two hypotheses justify the use of aggregated variables. Appendix B presents the details of the derivation of the discrete-space model derived using these aggregate variables. The discrete-space 
model can also be seen as a space discretization of the continuous-space model. It is worth highlighting that the discrete-space model still presents the three main features of the original formulation: first, the model is truly dynamic; second, the decision agents behave strategically; third, the model incorporates spatial aspects. None of the main features of the original model has been removed after the introduction of the aggregated variables.

The discrete-space model reads as follows (see Appendix B for the details): The objective of player $i$ is to maximize the space averaged payoff

$$
\widetilde{J}_{i}\left(v_{1}, \ldots, v_{J}, \boldsymbol{p}^{0}\right)=\int_{0}^{\infty} e^{-\rho t} \widetilde{G}_{i}\left(v_{i}, p_{i}\right) \mathrm{d} t
$$

subject to the dynamics of the aggregated stock of pollution in each subregion $\left(p_{i}, i=1, \ldots, J\right)$ described by the following system of ordinary differential equations:

$$
m_{i} \frac{\mathrm{d} p_{i}}{\mathrm{~d} t}=\sum_{\substack{j=0 \\ j \neq i}}^{J} k_{i j}\left(p_{j}-p_{i}\right)-m_{i} c_{i} p_{i}+m_{i} F_{i}\left(v_{i}\right), \quad i=1, \ldots, J .
$$

The system is supplemented with the initial conditions given by

$$
p_{i}(0)=\frac{1}{m_{i}} \int_{\Omega_{i}} P_{0}(\boldsymbol{x}) \mathrm{d} \boldsymbol{x}:=p_{i}^{0}, \quad i=1, \ldots, J
$$

where $P_{0}(\boldsymbol{x})$ is the initial data in $(3)$, and $\boldsymbol{p}^{0}=\left[p_{1}^{0}, \ldots, p_{J}^{0}\right]^{T}$.

Remark 1. The information concerning the spatial relationships among agents (see (63) for details) can be condensed by means of the matrix of coefficients in the first term of the right hand side of (8). More precisely, let us define the matrix $K$ by

$$
K=\left(\begin{array}{cccc}
k_{11} & k_{12} & \ldots & k_{1 J} \\
k_{21} & k_{22} & \ldots & k_{2 J} \\
\ldots & \ldots & \ldots & \ldots \\
k_{J 1} & \ldots & k_{J J-1} & k_{J J}
\end{array}\right)
$$

Note that $k_{i j}=0$ if and only if $\partial_{i j}=\emptyset$. That is, $k_{i j}=0, j \neq i$, if there is no common boundary between subregions $\Omega_{i}$ and $\Omega_{j}$ and $k_{i j} \neq 0$ only if subregions $\Omega_{i}$ and $\Omega_{j}$ have a common boundary $\partial_{i j} \neq \emptyset$. Furthermore, $k_{i i}=-\sum_{j \neq i} k_{i j}$. Then, matrix $K$ defines a graph (weighted graph) with one node for each subregion $\Omega_{i}$ and one edge joining nodes $i$ and $j$ if and only if $\partial_{i j} \neq \emptyset$ (see next section for some examples). This graph constitutes a simplified description of the geography of the region $\Omega$ and put more emphasis on interaction among neighbourhoods than on particular attributes, as for example, the size of regions or the length of common boundaries. These features have already been taken into account in the definition of the aggregate variables. The size of $k_{i j}$ indicates how fast the pollution spreads across $\partial_{i j}$ in absence of external transport phenomena. 
It is worth noting that $(7)$ and (8) define a $J$-player infinite horizon differential game with one decision variable for each player (the averaged emission rates in his subregion) and $J$ state variables (the averaged stock of pollution in each subregion) with time evolution described by the system of ODEs in (8). The first term in the right hand side of this differential equation collects the diffusion effect that tends to equilibrate the pollution between regions: the pollution entering $\Omega_{i}$ is proportional to the difference between the stock of pollution in the adjacent subregions. The pollution moves from subregions with high levels of concentration to subregions with low levels of concentration (Flick's law of diffusion). The second term is pollution degradability or natural degradation of the pollution stock. Finally, the third term is the flow of emissions.

We look for stationary Markov-perfect Nash equilibria of the differential game defined by (7)-(8). A $J$-tuple of admissible strategies $\Lambda^{*}=\left[\Lambda_{1}^{*}, \ldots, \Lambda_{J}^{*}\right]^{T}$ are a Markov-perfect Nash equilibrium if for all initial state $\boldsymbol{p}_{0}$,

$$
\widetilde{J}_{i}\left(\boldsymbol{v}^{*}, \boldsymbol{p}_{0}\right) \geq \widetilde{J}_{i}\left(\left[v_{i}, \boldsymbol{v}_{-i}^{*}\right], \boldsymbol{p}_{0}\right)
$$

for all admissible $v_{i}, i=1, \ldots, J$, where $\boldsymbol{v}^{*}=\left[v_{1}^{*}, \ldots, v_{J}^{*}\right]^{T}, v_{j}^{*}(t)=\Lambda_{j}^{*}\left(\boldsymbol{p}^{*}(t)\right), \boldsymbol{p}^{*}$ is the solution of (8) with $\boldsymbol{v}=\boldsymbol{v}^{*}$ and $\left[v_{i}, \boldsymbol{v}_{-i}^{*}\right]=\left[v_{1}^{*}, \ldots, v_{i}, \ldots, v_{J}^{*}\right]$. At any point in time, the emission decision of an agent depends only on the states of the averaged stocks of pollution in each subregion. These stationary Markovian strategies do not require precommitment to a course of action overtime (Haurie et al. (2012)).

\section{A linear-quadratic specification. Some examples}

In this section we analyze different examples and provide numerical results that help us understand the spatial problem of transboundary pollution. The different examples have been chosen in order to illustrate, on the one hand, the effects of the introduction of the strategic behaviour on the equilibrium environmental policies; and on the other hand, the differences with respect to the results obtained using standard dynamic game models which disregard the spatial aspect. From now on for simplicity and without loss of generality we assume that in (3) $P_{b}=0$. The linear-quadratic specification is inspired in the literature of transboundary pollution dynamic games (Jørgensen et al. 2010). Specifically, this specification is inspired by the transboundary pollution game in the seminal papers by Dockner \& Long (1993) and Ploeg \& Zeeuw (1992). Let us consider the following functional specifications:

$$
\begin{aligned}
& F_{i}\left(v_{1}, \ldots, v_{J}\right):=\beta_{i} v_{i}, \quad g_{i}\left(v_{1}, \ldots, v_{J}, \boldsymbol{p}\right):=v_{i}\left(A_{i}-\frac{v_{i}}{2}\right)-\frac{\varphi_{i}}{2} p_{i}^{2} \\
& \boldsymbol{p}=\left[p_{1}, \ldots, p_{J}\right]^{T}, \quad v_{i}=v_{i}(\boldsymbol{p}), \quad m_{i}=m_{j}, \forall i, j=1, \ldots, J
\end{aligned}
$$


With these functional forms the problem player $i$ is facing consists in choosing his control variable $v_{i}$ in order to maximize

$$
\begin{aligned}
J_{i}\left(v_{1}, \ldots, v_{J}, \boldsymbol{p}_{0}\right) & =\int_{0}^{+\infty} e^{-\rho t}\left(v_{i}\left(A_{i}-\frac{v_{i}}{2}\right)-\frac{\varphi_{i}}{2} p_{i}^{2}\right) \mathrm{d} t \\
\text { subject to: } \quad \dot{p}_{i} & =\sum_{j=1}^{J} k_{i j} p_{j}-c_{i} p_{i}+\beta_{i} v_{i}, \quad i=1, \ldots, J
\end{aligned}
$$

First, we focus on the two-player (two-region ${ }^{1}$ ) version of this differential game for which we can analytically characterized the feedback Nash equilibrium. Second, we consider more than two regions and numerically characterize the feedback Nash equilibrium of the differential game using the numerical method described in Appendix C.

\subsection{Two regions scenario}

Next proposition characterizes the feedback Nash equilibrium of the differential game with two players.

Proposition 1. Assuming interior solutions, the following vector of strategies

$$
v_{l}\left(p_{1}, p_{2}\right)=A_{l}+\beta_{l} \frac{\partial W_{l}}{\partial p_{l}}\left(p_{1}, p_{2}\right)=A_{l}+\beta_{l}\left(b_{l l}+a_{l l} p_{l}+e_{l} p_{3-l}\right), \quad l=1,2
$$

constitutes a Markov-perfect Nash equilibrium of the pollution game (10)-(11) with $J=2$, where $W_{i}\left(p_{1}, p_{2}\right)$ denotes region $i$ 's value function, given by:

$$
W_{i}\left(p_{i}, p_{j}\right)=\frac{1}{2} a_{i i} p_{i}^{2}+b_{i i} p_{i}+d_{i}+\frac{1}{2} a_{i j} p_{j}^{2}+b_{i j} p_{j}+e_{i} p_{i} p_{j}, \quad i, j=1,2, i \neq j .
$$

Constants $a_{i i}, b_{i i}, d_{i}, a_{i j}, b_{i j}, e_{i}, i, j=1,2, i \neq j$ satisfy the following system of twelve algebraic Riccati equations:

$$
\begin{aligned}
& a_{i i}^{2} \beta_{i}^{2}+2 e_{i}\left(k_{j i}+\beta_{j}^{2} e_{j}\right)-a_{i i}\left(2 c_{i}-2 k_{i i}+\rho\right)-\varphi_{i}=0, \\
& A_{i} a_{i i} \beta_{i}+A_{j} e_{i} \beta_{j}+b_{j j} e_{i} \beta_{j}^{2}+b_{i j}\left(k_{j i}+\beta_{j}^{2} e_{j}\right)-b_{i i}\left(c_{i}-k_{i i}-\beta_{i}^{2} a_{i i}+\rho\right)=0, \\
& \left(A_{i}+\beta_{i} b_{i i}\right)^{2}+2 \beta_{j} b_{i j}\left(A_{j}+\beta_{j} b_{j j}\right)-2 \rho d_{i}=0, \\
& e_{i}\left(2 k_{i j}+\beta_{i}^{2} e_{i}\right)-a_{i j}\left(2 c_{j}-2 k_{j j}-2 \beta_{j}^{2} a_{j j}+\rho\right)=0, \\
& \beta_{i} e_{i}\left(A_{i}+\beta_{i} b_{i i}\right)+\beta_{j} a_{i j}\left(A_{j}+\beta_{j} b_{j j}\right)+b_{i i} k_{i j}-b_{i j}\left(c_{j}-k_{j j}-\beta_{j}^{2} a_{j j}+\rho\right)=0, \\
& e_{i}\left(c_{i}+c_{j}-k_{i i}-k_{j j}-a_{i i} \beta_{i}^{2}-a_{j j} \beta_{j}^{2}+\rho\right)-a_{i i} k_{i j}-a_{i j}\left(k_{j i}+\beta_{j}^{2} e_{j}\right)=0,
\end{aligned}
$$

$i, j=1,2, i \neq j$.

\footnotetext{
${ }^{1}$ From now on and for simplicity in the notation we will use the word region to denote $\Omega_{i}, i=1, \ldots, J$.
} 
Proof. The sufficient condition for a stationary feedback Nash equilibrium requires us to find bounded and continuously differentiable functions, denoted by $W_{i}\left(p_{1}, p_{2}\right)$, which satisfy, for all $p_{1}(t) \geq 0, p_{2}(t) \geq 0$ the Hamilton-Jacobi-Bellman (HJB) equations for the two players. We first concentrate on finding solutions for the HJB equations. These equations are given by

$$
\begin{aligned}
\rho W_{i}\left(p_{i}, p_{j}\right)= & \max _{v_{i} \geq 0}\left[v_{i}\left(A_{i}-\frac{v_{i}}{2}\right)-\frac{\varphi_{i}}{2} p_{i}^{2}+\frac{\partial W_{i}}{\partial p_{i}}\left(p_{i}, p_{j}\right)\left(k_{i j} p_{j}+k_{i i} p_{i}-c_{i} p_{i}+\beta_{i} v_{i}\right)\right. \\
& \left.+\frac{\partial W_{i}}{\partial p_{j}}\left(p_{i}, p_{j}\right)\left(k_{j i} p_{i}+k_{j j} p_{j}-c_{j} p_{j}+\beta_{j} v_{j}\right)\right] .
\end{aligned}
$$

The first-order optimality conditions read:

$$
v_{i}\left(p_{i}, p_{j}\right)=A_{i}+\beta_{i} \frac{\partial W_{i}}{\partial p_{i}}\left(p_{i}, p_{j}\right)
$$

if these expressions are positive, and zero otherwise.

Guided by the model's linear-quadratic structure, we restrict our attention to linear strategies and assume that the players' value functions are quadratic and given by (13). Taking into account this specification of region $i$ 's value function in (13), the optimal emission strategies can be written as in expression (21).

Inserting (21) in (20), the six Riccati equations for each player that characterize the coefficients of the value functions $a_{i i}, b_{i i}, d_{i}, a_{i j}, b_{i j}, e_{i}, i, j=1,2, i \neq j$ are determined by identification.

A sufficient condition guaranteeing that the expression in (13) is region $i$ 's value function, and that (21) are the emission strategies is given by

$$
\lim _{t \rightarrow \infty} e^{-\rho t} W_{i}\left(p_{1}(t), p_{2}(t)\right)=0,
$$

where $\left(p_{1}(t), p_{2}(t)\right)$ is the solution of the closed-loop dynamics obtained after substituting the optimal emission strategies (21) into the pollution stock dynamics given by (11).

This solution can be written as $p_{1}(t)=C_{1} e^{\lambda_{1} t}+C_{2} e^{\lambda_{2} t}+p_{1}^{S S}, p_{2}(t)=C_{3} e^{\lambda_{1} t}+C_{4} e^{\lambda_{2} t}+p_{2}^{S S}$, where $p_{i}^{S S}$ refers to the steady state of the pollution stock in region $i, C_{i}, i=1, \ldots, 4$, are constants and $\lambda_{i}, i=1,2$ are the real eigenvalues of the matrix associated to the system of linear differential equations.

The functional specification in (13) allows condition (22) to be satisfied whenever the pollution stocks are bounded. This condition is guaranteed if the steady state is globally asymptotically stable.

In order to focus on the spatial aspect of the pollution game, we assume

$$
\beta_{i}=\beta, \varphi_{i}=\varphi, A_{i}=A, \quad c_{i}=c, i=1,2 .
$$


These conditions imply that the players are completely symmetrical in every respect, except in their geographical positions described by the entries $k_{i j}$ of matrix $K$ (see Remark 1 ). The only exception is Example 1 in which the position of both regions is completely interchangeable.

Next two examples completely characterize the emission equilibrium strategies making use of the algebraic Riccati equations presented in Proposition 1.

\subsubsection{Example 1: Two regions isolated from outside}

Let us start with the simplest possible configuration corresponding to the case of two identical regions (players) with identical geographical position. Player $i$ controls the averaged emissions in his own region $\Omega_{i}$. The two regions are interconnected (there is one edge joining regions $\Omega_{1}$ and $\Omega_{2}$, each region represented by one node) and are isolated from outside. That is, there is no flux of pollution neither entering nor exiting the geographical space formed by the two regions $\left(k_{10}=k_{20}=0\right)$. To gain intuition we can think on a groundwater reservoir shared by two neighbouring regions. The agricultural activities carried out in both regions contribute to the pollution of the reservoir due to pesticides or other contaminants as, for example, phosphore. Another example could be a lake polluted by wastewater produced by economic activities located in two different regions. These problems are certainly relevant (there are more than 300 transboundary aquifer systems worldwide, UN-Water Thematic Paper (2008)). We will use these particular applications as a guide to gain intuition about the type of problems that can be analyzed with the techniques described in this paper. In this first example, the aquifer (the groundwater reservoir or the lake) is isolated from other sources or sinks of pollution.

The following figure represents this situation. On the left, a schematic representation of the position of regions $\Omega_{1}$ and $\Omega_{2}$ is shown. This sketch can be condensed straightforwardly in the graph represented in the middle of the figure, with $K$ the matrix containing the spatial relationship among the two regions, and $k_{12}$ indicating how fast pollution spreads across the common boundary between $\Omega_{1}$ and $\Omega_{2}$.
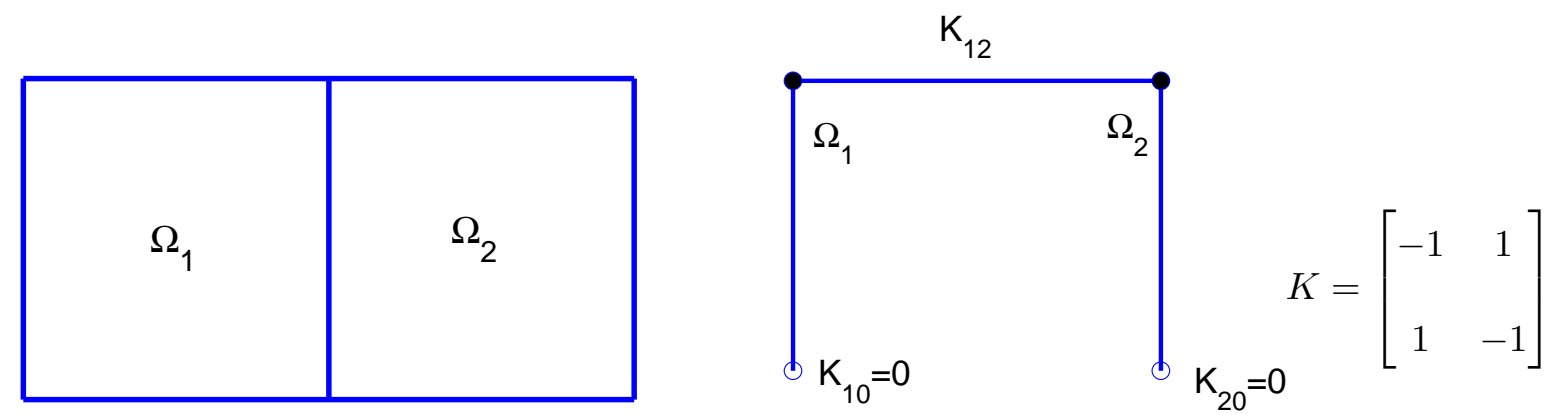

The completely symmetric spatial relationship among regions $\Omega_{1}$ and $\Omega_{2}$ (a symmetric matrix $K$ ) 
allows us to look for symmetric emission strategies and symmetric value functions. Therefore, under the following conditions must be hold $a_{j j}=a_{i i}, b_{j j}=b_{i i}, a_{j i}=a_{i j}, b_{j i}=b_{i j}, e_{j}=e_{i}, d_{j}=d_{i}$. Therefore, the system of twelve algebraic Ricatti equation (14)-(19) reduces to six equations with unknowns $a_{i i}, b_{i i}, a_{i j}, b_{i j}, e_{i}, d_{i}$. From (15)-(19) the following expressions can be easily derived:

$$
\begin{aligned}
b_{i i} & =\frac{b_{i j}+A \beta\left(a_{i i}+e_{i}\right)+\beta^{2} b_{i j} e_{i}}{1+c-\beta^{2} a_{i i}-\beta^{2} e_{i}+\rho}, \\
d_{i} & =\frac{\left(A+\beta b_{i i}\right)\left(A+\beta b_{i i}+2 \beta b_{i j}\right)}{2 \rho}, \\
a_{i j} & =\frac{\left(2+\beta^{2} e_{i}\right) e_{i}}{2(1+c)-2 \beta^{2} a_{i i}+\rho}, \\
b_{i j} & =\frac{A \beta\left(a_{i j}+e_{i}\right)+b_{i i}\left(1+\beta^{2} a_{i j}+\beta^{2} e_{i}\right)}{1+c-\beta^{2} a_{i i}+\rho}, \\
e_{i} & =\frac{a_{i i}+a_{i j}}{2(1+c)-2 \beta^{2} a_{i i}-\beta^{2} a_{i j}+\rho} .
\end{aligned}
$$

Substituting (26) in (28), one gets a first equation relating coefficients $a_{i i}$ and $e_{i}$ :

$$
2 a_{i i}^{2} \beta^{2}\left(1+2 \beta^{2} e_{i}\right)-a_{i i}\left(1+4 \beta^{2} e_{i}\right)(2+2 c+\rho)+\left(2+4 c^{2}-3 e_{i} \beta^{2}\right) e_{i}-\beta^{4} e_{i}^{2}+4 \rho+\rho^{2}+4 c(2+\rho)=0 .
$$

This equation together with (14) constitutes a system of two non-linear equations for coefficients $a_{i i}$ and $e_{i}$. With Mathematica 11.0 we solve this system for the following parameter values

$$
\beta=1, \quad \varphi=1, \quad A=0.5, \quad \rho=0.01, \quad c=0.5
$$

Unless otherwise indicated, these parameter values are used in all the numerical examples.

The system admits six possible solutions. Substituting (27) and (26) in (24), a new expression of $b_{i i}$ in terms of $a_{i i}$ and $e_{i}$ can be easily derived. Then, six possible values of $b_{i i}$ are obtained. Once coefficients $a_{i i}, e_{i}$ and $b_{i i}$ are known, the feedback optimal strategies are completely characterized. Only one of the six possible solutions for $a_{i i}$ and $e_{i}$ implies two negative eigenvalues of the matrix associated to the closed-loop dynamical system obtained after substituting the optimal emission strategies. This solution is given by: $a_{i i}=-0.354746, e_{i}=-0.108611$ and correspondingly $b_{i i}=-0.172108$. From (26), (27) and (25), $a_{i j}=-0.0552293, b_{i j}=-0.121105, d_{i}=1.40474$.

Therefore, the optimal emission strategies read:

$$
\left[\begin{array}{l}
v_{1}\left(p_{1}, p_{2}\right) \\
v_{2}\left(p_{1}, p_{2}\right)
\end{array}\right]=L\left[\begin{array}{l}
p_{1} \\
p_{2}
\end{array}\right]+\left[\begin{array}{l}
0.32798 \\
0.32798
\end{array}\right] \text { where } L=\left[\begin{array}{ll}
-0.35475 & -0.10861 \\
-0.10861 & -0.35475
\end{array}\right] .
$$

Functions $v_{1}$ and $v_{2}$ are the feedback Nash equilibrium strategies for player 1 and 2, respectively. In this example there is no difference between the regions from a spatial (geographical) point of view. 

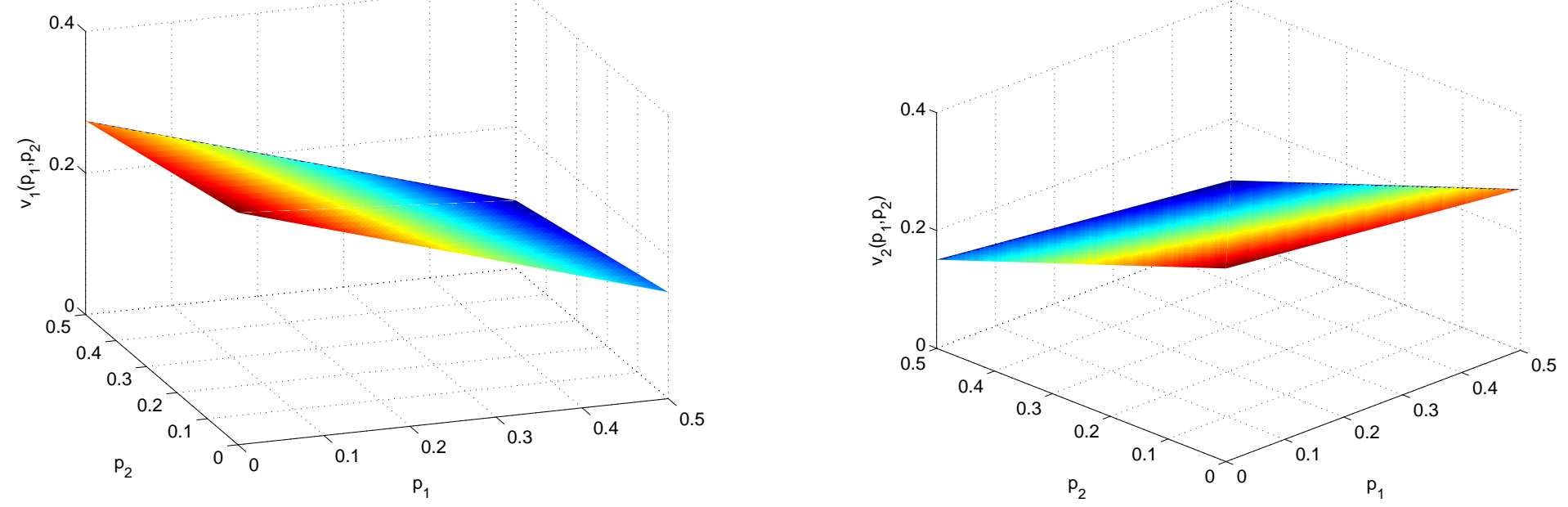

Figure 1: Example 1. Regions' feedback strategies. Region 1 (left); Region 2 (right)

Figure 1 left and right shows the feedback Nash equilibrium strategies for player 1 and 2, respectively. These strategies are the equilibrium emissions as functions of the two state variables, the pollution stocks in regions 1 and $2\left(p_{1}\right.$ and $\left.p_{2}\right)$. The feedback rule in each region negatively depends on the pollution stock accumulated in both regions. As expected the marginal effect of an increase of the own pollution stock is greater (in absolute terms) than the marginal effect of an increase of the pollution stock accumulated in the other region. Each region partially internalizes to a greater extent the damages caused by its own pollution stock than those caused by the pollution stock of its neighbouring region. This is due to the fact that the environmental damage function only depends on the pollution stock accumulated in its own region. Furthermore, due to the completely symmetric character of this scenario, the role played by $p_{i}$ and $p_{j}, j \neq i$ in the optimal strategy of player $i$ is identical to the role played by $p_{j}$ and $p_{i}, i \neq j$ in the optimal strategy of player $j$. That is, the feedback strategies satisfy the symmetry condition $v_{1}\left(p_{1}, p_{2}\right)=v_{2}\left(p_{2}, p_{1}\right)$.

The identical steady-state values of the pollution stocks are $p_{1}^{S S}=p_{2}^{S S}=0.340365$, and the time paths of the pollution stock along the equilibrium strategy are given by

$$
\begin{aligned}
& p_{1}(t)=\left(\left(p_{1}(0)-p_{1}^{S S}\right)-\left(p_{2}(0)-p_{2}^{S S}\right)\right) e^{\lambda_{1} t} / 2+\left(\left(p_{1}(0)-p_{1}^{S S}\right)+\left(p_{2}(0)-p_{2}^{S S}\right)\right) e^{\lambda_{2} t} / 2+p_{1}^{S S}, \\
& p_{2}(t)=\left(\left(p_{2}(0)-p_{2}^{S S}\right)-\left(p_{1}(0)-p_{1}^{S S}\right)\right) e^{\lambda_{1} t} / 2+\left(\left(p_{1}(0)-p_{1}^{S S}\right)+\left(p_{2}(0)-p_{2}^{S S}\right)\right) e^{\lambda_{2} t} / 2+p_{2}^{S S},
\end{aligned}
$$

where $\lambda_{1}=-2.7461$ and $\lambda_{2}=-0.96336$ are the eigenvalues of the matrix $H=K-c I+L$.

The symmetric behavior clearly appears when drawing the time-paths of the emission levels and the pollution stock along the equilibrium strategy if the initial state is identical for both players $p_{1}(0)=$ $p_{2}(0)$. In Figure 2 for illustration purposes the particular case $p_{i}(0)=0.1, i=1,2$ is considered. The plot on the right shows the optimal time-paths of the pollution stocks that are, as expected, identical 

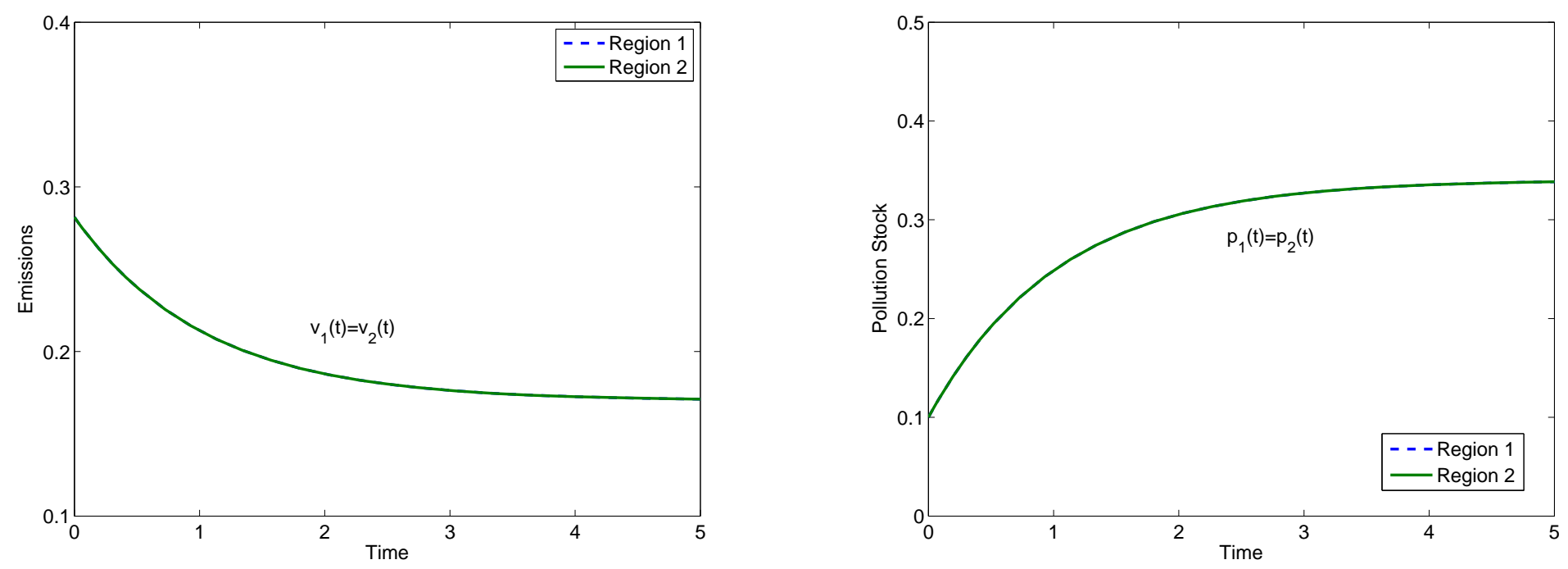

Figure 2: Example 1. Emission (left) and pollution stock (right) time-paths along the equilibrium strategy

in both regions. From the initial value 0.1 along the optimal time-path the stock of pollution increases towards its steady state value. Correspondingly, the time-path of emissions along the equilibrium strategy, on the left of Figure 2, coincides for both players and, as expected, decreases with time until the steady-state value is attained.

\subsubsection{Example 2: Two regions with different geographical neighbourhoods}

Let us now introduce in the two-player setting a unique asymmetric factor related to the geographical position. The two regions are again interconnected, but unlike the previous example only region 1 is isolated from outside $\left(k_{10}=0\right)$. Region 2 shares a boundary $\left(k_{20}=1\right)$ with another very large region with a lower concentration of pollutants than region 2. This external region acts a sink of pollution. In particular, as an extreme case, here we assume that the pollution stock in this additional region is zero $\left(p_{20}=0\right)$. With this geographical configuration there is a flux of pollution from region 2 to the pollution sink, but not in the opposite direction. Using the same application of Example 1, we can think on a part of the aquifer that is not affected by human activities and shares boundary with region 2. This clean part of the aquifer is large enough to absorb pollutants with negligible increment of the concentration of pollutants. With this example we try to understand in a simple configuration the spatial effects that can appear in the strategic behaviour of agents when geographical considerations are incorporated to common transboundary pollution games.

The figure represents such a situation. In the schematic representation on the left we have marked with a dashed line the part of the boundary of region 2 in contact with the pollution sink. The graph and matrix $K$ condense the same information: 

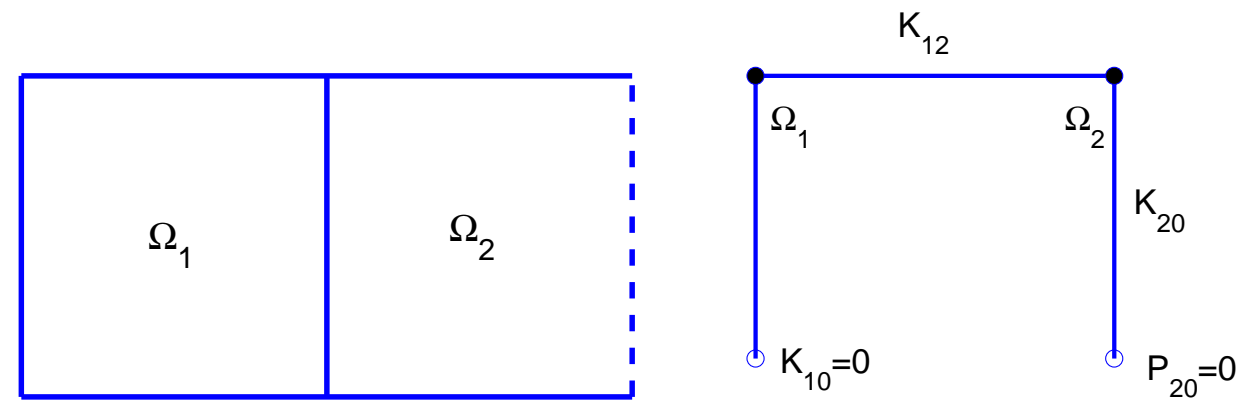

$K=\left[\begin{array}{cc}-1 & 1 \\ 1 & -2\end{array}\right]$

In this scenario we have to deal with a system of twelve algebraic Ricatti equation given by (14)-(19). Under assumption (23), from equation (19) for player $i$ and $j$, respectively, one gets:

$$
e_{i}=\frac{a_{i i}+a_{i j}\left(1+\beta^{2} e_{j}\right)}{3+2 c-\beta^{2} a_{i i}-\beta^{2} a_{j j}+\rho}, \quad e_{j}=\frac{a_{j j}+a_{j i}\left(1+\beta^{2} e_{i}\right)}{3+2 c-\beta^{2} a_{i i}-\beta^{2} a_{j j}+\rho} .
$$

Equation (17) for player $i$ and equation (14) for player $j$, respectively, lead to:

$$
a_{i j}=\frac{e_{i}\left(2+\beta^{2} e_{i}\right)}{2(2+c)-2 \beta^{2} a_{j j}+\rho}, \quad a_{j i}=\frac{e_{j}\left(2+\beta^{2} e_{j}\right)}{2(1+c)-2 \beta^{2} a_{i i}+\rho} .
$$

Substituting (33) in (32) one gets two equations relating coefficients $a_{i i}, a_{j j}, e_{i}$ and $e_{j}$ :

$$
\begin{aligned}
& e_{i}\left(2+\beta^{2} e_{i}\right)\left(1+\beta^{2} e_{j}\right)+\left(a_{i i}-e_{i}\left(3+2 c-\beta^{2} a_{i i}-\beta^{2} a_{j j}+\rho\right)\right)\left(4+2 c-2 \beta^{2} a_{j j}+\rho\right)=0, \\
& e_{j}\left(2+\beta^{2} e_{j}\right)\left(1+\beta^{2} e_{i}\right)+\left(a_{j j}-e_{j}\left(3+2 c-\beta^{2} a_{i i}-\beta^{2} a_{j j}+\rho\right)\right)\left(2+2 c-2 \beta^{2} a_{i i}+\rho\right)=0 .
\end{aligned}
$$

These two equations together with equation (14) for player $i$ and equation (17) for player $j$

$$
\beta^{2} a_{i i}^{2}+2 e_{i}\left(1+\beta^{2} e_{j}\right)-(2+2 c+\rho) a_{i i}-\varphi=0, \quad \beta^{2} a_{j j}^{2}+2 e_{j}\left(1+\beta^{2} e_{i}\right)-(4+2 c+\rho) a_{j j}-\varphi=0,
$$

define a system of four nonlinear equations for coefficients $a_{i i}, a_{j j}, e_{i}$ and $e_{j}$. This system is numerically solve using the parameter values in (29). From the multiple solutions the unique associated with a globally stable steady-state reads $a_{i i}=-0.344173, a_{j j}=-0.209672, e_{i}=-0.081391, e_{j}=-0.051392$.

Once the values of $a_{i i}, a_{j j}, e_{i}$ and $e_{j}$ are known, from equations (15), (17) and (18) for player $i$ and equations (14), (15) and (18) for player $j$, a system of two linear equations for coefficients $b_{i i}$ and $b_{j j}$ can be obtained. The solution is $b_{i i}=-0.147536, b_{j j}=-0.062412$.

From (33), equations (16) and (18) for player $i$ and equations (15) and (16) for player $j: a_{i j}=$ $-0.0287618, a_{j i}=-0.0270778, b_{i j}=-0.0694238, b_{j i}=-0.0509362, d_{i}=3.17362, d_{j}=7.77885$.

Therefore, the optimal emission strategies read:

$$
\left[\begin{array}{l}
v_{1}\left(p_{1}, p_{2}\right) \\
v_{2}\left(p_{1}, p_{2}\right)
\end{array}\right]=L\left[\begin{array}{l}
p_{1} \\
p_{2}
\end{array}\right]+\left[\begin{array}{l}
0.352463 \\
0.437588
\end{array}\right] \text { where } L=\left[\begin{array}{cc}
-0.344173 & -0.0813912 \\
-0.051392 & -0.209672
\end{array}\right]
$$


These functions are the feedback Nash equilibrium strategies for player 1 and 2, respectively. As in the previous example, both equilibrium strategies negatively depend on both pollution stocks, being more sensitive to changes in its own pollution stock than to changes in the pollution stock of its neighbour. The feedback strategies have lost the symmetric property presented in Example 1 (Figure 1) as Figure 3 shows. The influence of the geographical position in the feedback Nash equilibrium strategies is evident.
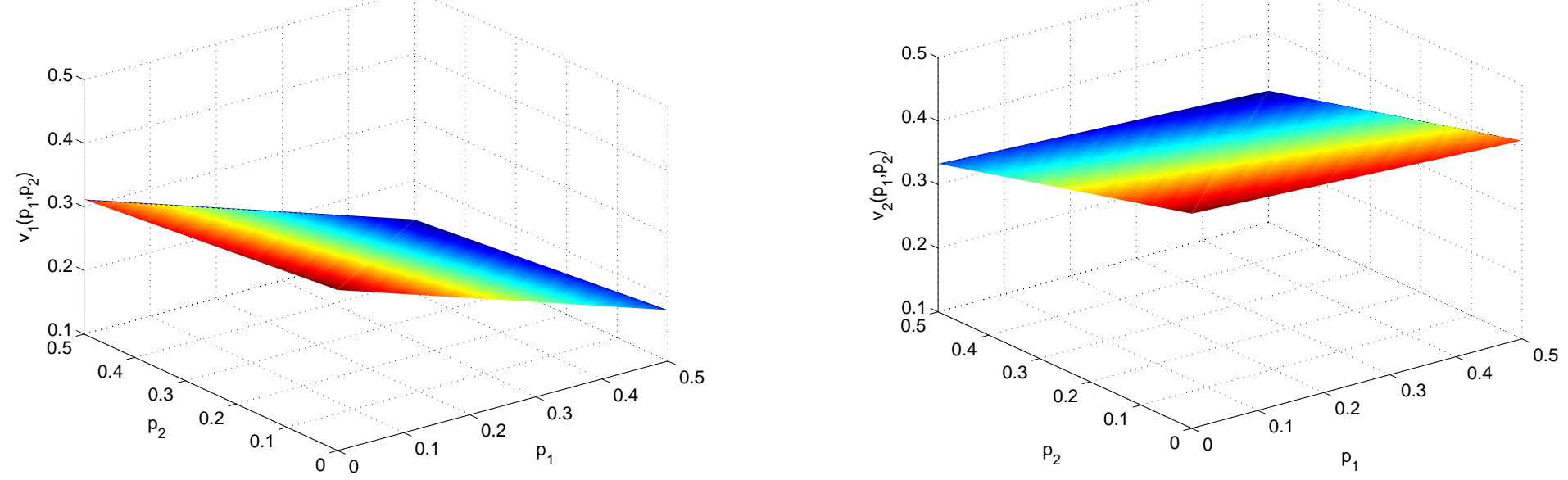

Figure 3: Example 2. Regions' feedback strategies. Region 1 (left); Region 2 (right)

The steady-state values of the pollution stocks are $p_{1}^{S S}=0.328921, p_{2}^{S S}=0.276641$, and the time paths of the pollution stock along the equilibrium strategy are given by

$$
\begin{array}{r}
p_{1}(t)=p_{1}^{S S}+\left(0.446396\left(p_{2}(0)-p_{2}^{S S}\right)+0.710294\left(p_{1}(0)-p_{1}^{S S}\right)\right) e^{\lambda_{1} t} \\
-\left(0.446396\left(p_{2}(0)-p_{2}^{S S}\right)-0.289706\left(p_{1}(0)-p_{1}^{S S}\right)\right) e^{\lambda_{2} t}, \\
p_{2}(t)=p_{2}^{S S}+\left(0.289706\left(p_{2}(0)-p_{2}^{S S}\right)+0.460974\left(p_{1}(0)-p_{1}^{S S}\right)\right) e^{\lambda_{1} t} \\
+\left(0.710294\left(p_{2}(0)-p_{2}^{S S}\right)-0.460974\left(p_{1}(0)-p_{1}^{S S}\right)\right) e^{\lambda_{2} t},
\end{array}
$$

where $\lambda_{1}=-1.24801$ and $\lambda_{2}=-3.30584$ are the eigenvalues of the matrix $H=K-c I+L$.

Again the initial pollution stocks in each region are assumed to be both equal to 0.1. Then, the only difference between the two regions is their geographical position.

On the other hand, more importantly, as Figure 4 shows, region 2 can benefit from its spatial position and emit along the equilibrium strategy above the level of emission of region 1. However, interestingly, the time path of the stock of pollution in region 2 along the equilibrium strategy is always lower than the corresponding stock of pollution in region 1 . The sole reason for this behaviour is that there is an important flow of pollution exiting region 2 towards the boundary region $\Omega_{0}$ (the sea). This allows region 2 to considerably increase its emissions in comparison with those of region 1 that due to its geographical position only can "exchange" pollution concentration with region 2. Because the latter is 

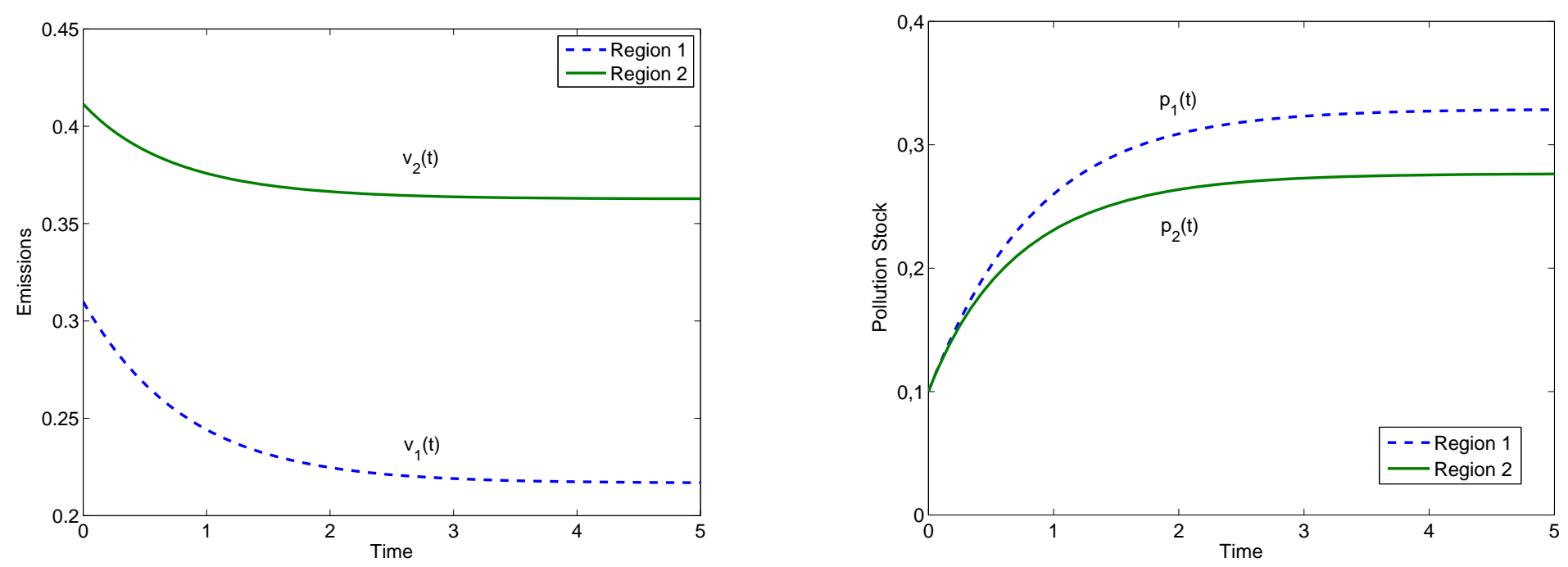

Figure 4: Example 2. Emission (left) and pollution stock (right) time-paths along the equilibrium strategy

emitting at a high emission rate, its pollution concentration is high and in consequence, there is a net flow of pollution moving from region 2 to region 1. It is worth noting that the somehow counterintuitive result "the higher the emissions, the lower the pollution stock" is due exclusively to the inclusion of the spatial aspect in the model at hand. This result stresses the importance of taking into account this aspect and cannot be reproduced in a transboundary pollution dynamic game with symmetric players when the spatial transport of pollution is neglected.

\subsection{More than two regions scenario}

In this section feedback Nash equilibria of the differential game (10)-(11) with more than two players are numerically characterized. In the two-region scenario and for the ease of presentation we have focussed only on interior Markov-perfect Nash equilibrium avoiding the analysis of corner solutions. The numerical method described in Appendix $\mathrm{C}$ allows us to characterize the feedback rules as piecewise affine functions that combine strictly positive emission rates for some values of the pollution stocks together with zero emission levels for greater values of the pollution stock. The two-region examples have served as tests to verify the convergence of the numerical algorithm to the appropriate equilibrium. We have checked that as long as the emission rates are positive the analytically and the numerically computed strategies coincide up to a small numerical error. 


\subsubsection{Example 3: Three regions isolated from outside}

Let us consider three regions and the geographical configuration described by the following schema with matrix $K$ containing the spatial relationships among these regions.
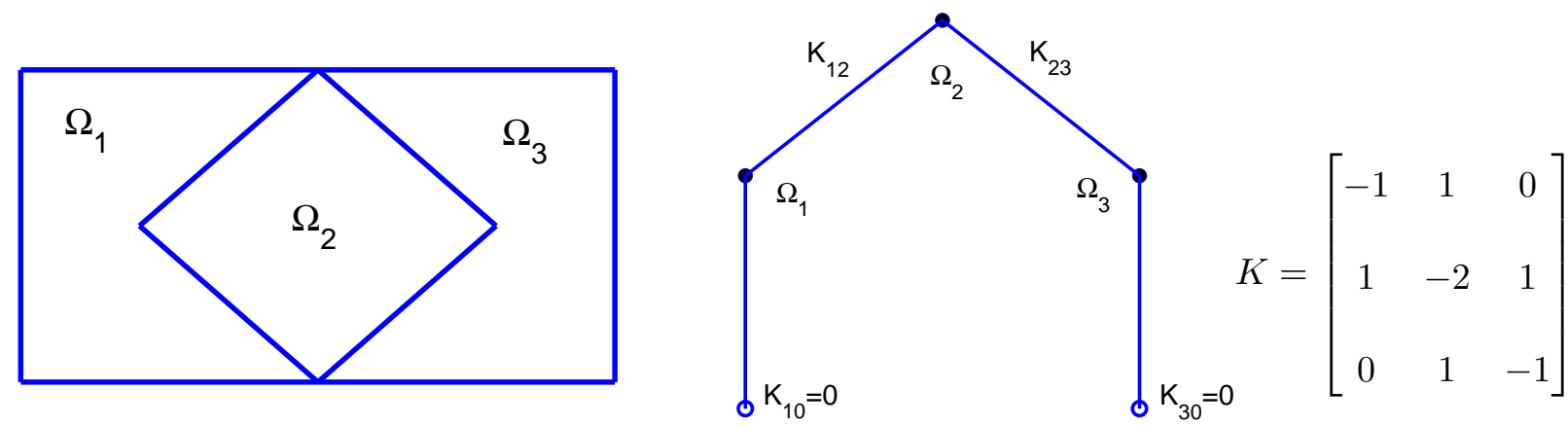

As in Example 1 the three regions are isolated from outside $\left(k_{10}=k_{30}=0\right)$, but unlike Example 1 the regions are not all identical with respect to their spatial position. Specifically, regions 1 and 3 are identical and share boundary with region 2 and with the exterior of the region under consideration. In this last case without exchange neither in nor out of pollution. Region 2 has a different geographical position because it shares boundary with regions 1 and 3 . This configuration can be applied in the same context as in Example 1 but this time with the three regions polluting the same aquifer.
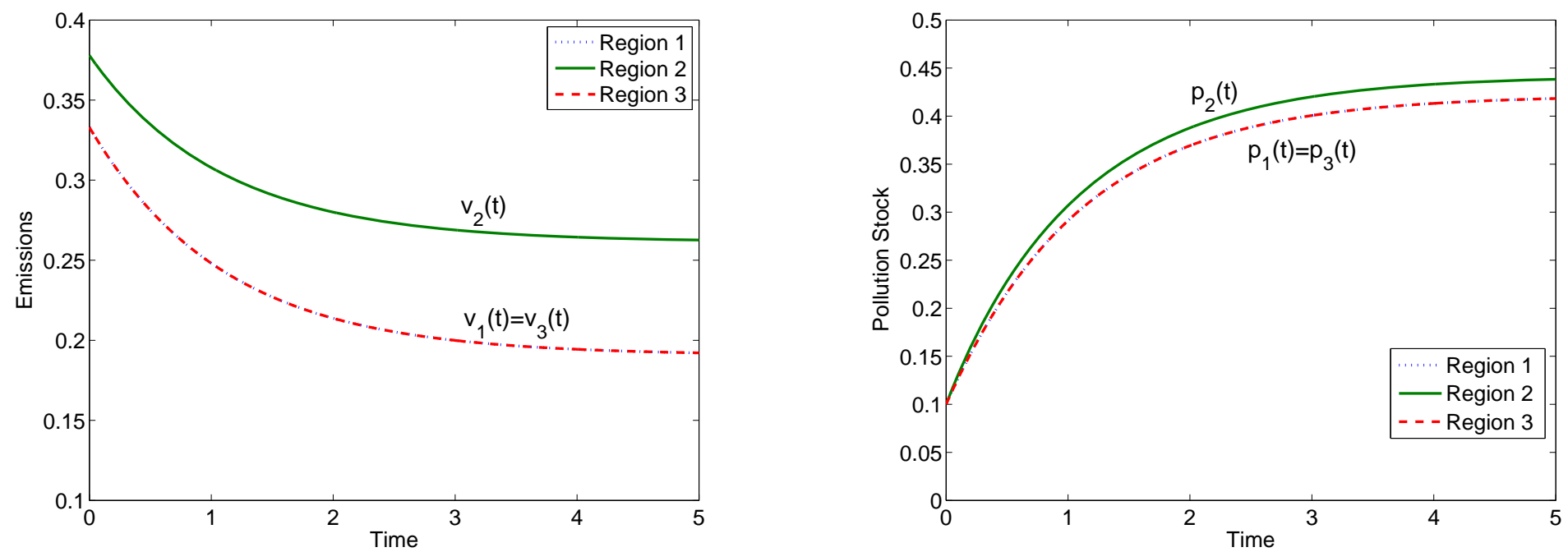

Figure 5: Example 3, $p_{i}(0)=0.1$. Emission (left) and pollution stock (right) time-paths along the equilibrium strategy

We have computed the feedback Nash equilibrium strategies that, for this example, are functions of three state variables (the pollution stock in each region). We refrain from drawing these strategies, and we just draw the time-paths of emissions and pollution stock along the equilibrium strategy. Figure 5 
shows only two curves in each graph, because, as expected, the level of emissions in regions 1 and 3 are equal, and consequently, the optimal time-path of the pollution stock coincides for both regions. Unlike in Example 2, in the present example the region with a higher level of emissions (region 2) presents a higher stock of pollution too. This is due to the spatial positions of the regions. Region 1 (the same argument applies to region 3) has to reduce its equilibrium emission to attain its pollution steady-state value. Because of its geographical position, region 1 only can exchange pollution with region 2 , that in this case has a greater concentration of pollution because it receives from the other two regions. As a result, the pollution flows from the region with a greater concentration (region 2) to the region with a lower concentration (region 1 ).
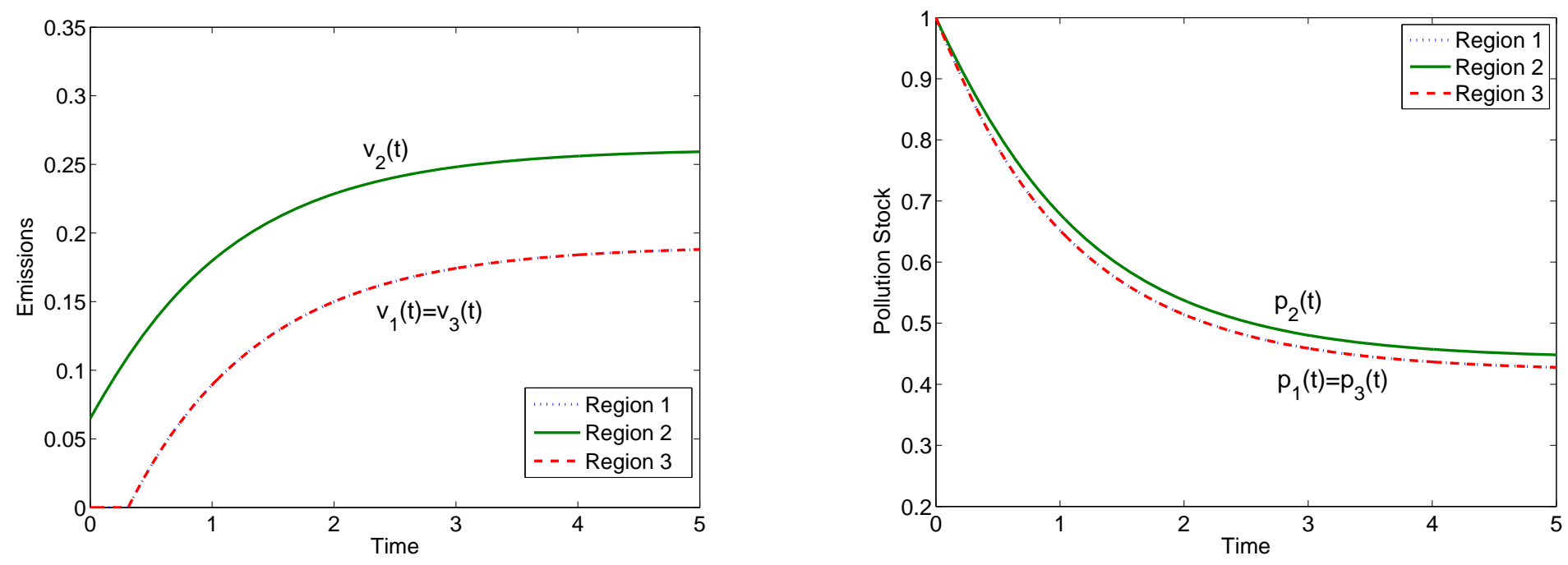

Figure 6: Example 3, $p_{i}(0)=1$. Emission (left) and pollution stock (right) time-paths along the equilibrium strategy

Figure 6 presents for this example the emission and pollution stock time-paths along the equilibrium strategy when the initial pollution stocks are assumed to be equal to 1 in the three regions. As expected, with high initial pollution stocks the optimal time-paths decrease towards their steady-state levels (Figure 6 right). The initially high level of the stock of pollution prevents regions 1 and 3 emit until the level of pollution drops below a certain threshold (Figure 6 left). After this initial period of time, the emission rates increase towards their long-run values.

\subsubsection{Example 4: Four regions isolated from outside}

Let us consider four regions and the geographical configuration described by the following schema 

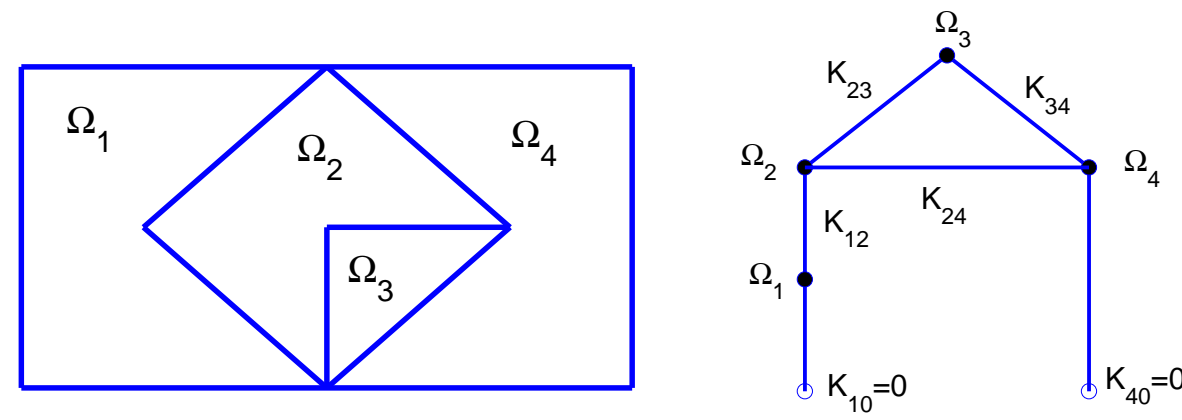

$K=\left[\begin{array}{cccc}-1 & 1 & 0 & 0 \\ 1 & -3 & 1 & 1 \\ 0 & 1 & -2 & 1 \\ 0 & 1 & 1 & -2\end{array}\right]$

This configuration can be applied in the same context as in Example 1 but this time with the four regions polluting the same aquifer. As the previous graph shows, the four regions as a whole are isolated from outside $\left(k_{10}=k_{40}=0\right)$. In this spatial configuration region 1 shares boundaries with region 2 and the exterior of the whole region under consideration, $\Omega$, but no exchange of pollution is possible. Region 2 shares boundary with the other three regions, while region 3 has a common boundary with regions 2 and 4. Finally, region 4 shares boundary with regions 2 and 3, as well as with the exterior of the whole region $\Omega$, from which it is isolated. Therefore, regions 3 and 4 are identical in terms of the flow of pollution concentration, while regions 1 and 2 are different from each other and different from the other two regions. It is worth noting that this example is genuinely two dimensional in the sense that the particular spatial configuration showed in this example cannot be reproduced in a one-dimensional setting.
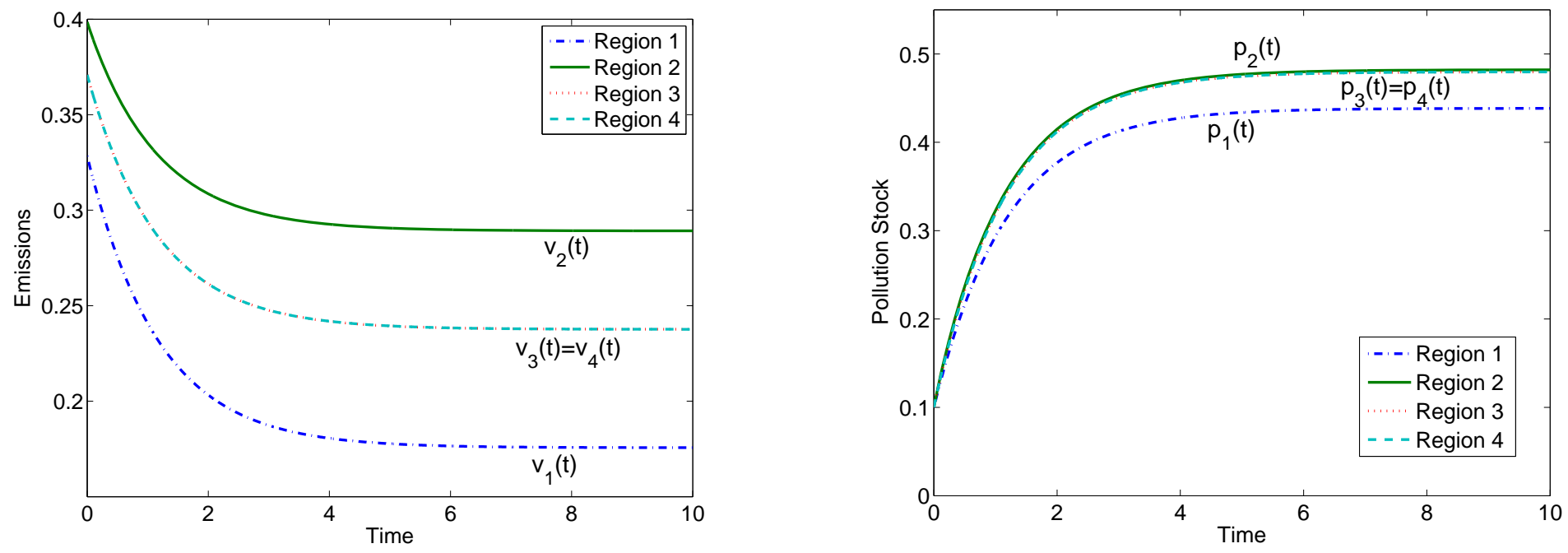

Figure 7: Example 4. Emission (left) and pollution stock (right) time-paths along the equilibrium strategy

Figure 7 clearly illustrates the geographical advantage of region 2. Because this region has three neighbours to which the pollution can flow, along the equilibrium strategy it can emit at a rate clearly 
greater than that of regions 3 and 4 at each instant of time (Figure 7 left). However, the optimal time-path of the stock of pollution is similar for all these three regions (Figure 7 right).

\subsubsection{Example 5: Four regions with different geographical neighbourhoods}

Let us consider now an example quite similar to the previous one, but presenting two main differences, as shown in the following schema:


Firstly, unlike the previous example only region 1 is isolated from outside $\left(k_{10}=0\right)$. Region 4 shares a boundary with the exterior of the whole region $\Omega$ which is assumed to have a lower (zero) concentration of pollutants than region $4\left(p_{40}=0\right)$. Secondly, the natural regeneration rate $c_{i}$ is assumed to be zero in the four regions $\left(c_{i}=0\right)$. This configuration can be applied in the same context as in Example 2 but this time with the four regions polluting the same aquifer with only region 4 sharing a boundary with the pollution sink.
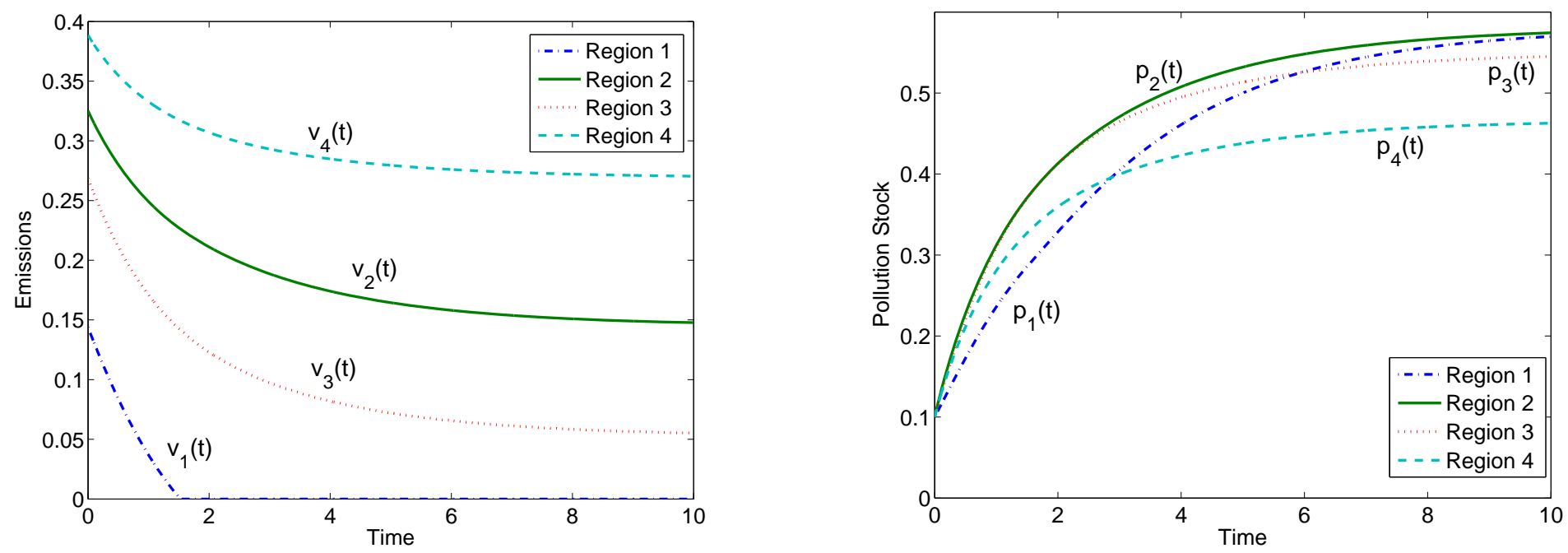

Figure 8: Example 5. Emission (left) and pollution stock (right) time-paths along the equilibrium strategy

As Figure 8 shows the region emitting at the highest level (region 4) along the whole time horizon 
attains the lowest stock of pollution in the long run. As in Example 2 this in principle counterintuitive result comes as a result that there is a flow of pollution from region 4 to outside $\Omega$, while no flow is entering region 4 from outside $\Omega$. Because the stock of pollution is assumed to be zero outside $\Omega$, there is an important flow of pollution exiting region 4 towards the exterior of the whole region $\Omega$. This fact allows region 4 considerably increase its emissions in comparison with those of the other regions that because of their geographical positions only can "exchange" pollution concentration with regions in $\Omega$.

Figure 8 also shows that the steady-state values of the stock of pollution in regions 1,2 and 3 are quite similar, despite the fact that region 1 emits along the whole time horizon at a much softer level than the other regions. More important, region 1 does not emit at all after an initial period of time. The sole reason for this behaviour is that region 1 only can exchange pollution with region 2, while the other regions have two or three neighbours which can be the recipients of the flow of pollution.

It worth noting that contrary to what happens in the standard case, a differential game without spatial interactions, a positive natural regeneration of the environment is not needed in order to ensure the convergence of the optimal time-path of the pollution stock towards an asymptotically stable steadystate. However, the lack of natural degradation of the pollution stock could imply that one of the regions has to stop emitting during a period of time (as region 1 does in this example).

\subsection{Cooperative vs. non-cooperative strategies}

In this section we compare the cooperative and non-cooperative strategies of the transboundary pollution dynamic game with spatial effects to assess the impact of the strategic behaviour of the decision makers in the definition of the environmental policies. All the previous examples present the noncooperative strategies, the feedback Nash equilibrium strategies obtained assuming that each region's emission policy is chosen to further its own interest, given the other regions' emission policies. In the cooperative scenario a unique decision maker (an environmental regulator) chooses all regions' emission policies in order to maximize the joint welfare of all regions. Therefore, the cooperative solution is obtained solving a $J$-state variable optimal control problem. Any cooperative outcome constitutes a first-best solution but relies on a high degree of commitment to follow the agreed-upon emission policies.

First, we analytically characterize the cooperative solution for the two examples which correspond to a two-player specification. For these examples, we also propose an optimal taxation scheme as a possible environmental policy to sustain the cooperative Pareto efficient outcome ${ }^{2}$. Among the different environmental policy instruments usually used in the dynamic game literature (Jørgensen et al.

\footnotetext{
${ }^{2}$ We thank an anonymous reviewer of this journal for suggesting this analysis.
} 
(2010)), we focus on an intertemporal pollution taxation scheme which assumes perfect information as in Benchekroun \& Long (1998). We are concerned with efficiency-inducing taxation and prove that there exist time-independent tax rules that guide the regions to achieve the socially optimum time paths of emission and pollution. The optimal tax per unit of emission is dependent on the current level of the pollution stocks of both regions. The proposed linear Markov perfect tax rules ensure subgame perfection avoiding time inconsistency, and induce the regions to emit at the efficient emission levels.

We are aware that this type of scheme can be difficult to implement since it would require perfect monitoring by the environmental regulator. Other approaches based on taxes proposed in the literature to overcome this informational problem include to choose a fixed tax rate on loading (Mäler et al. (2003)) or to define schemes which take the form of charges per unit deviation between desired and observed pollutant accumulations paths (Xepapadeas (1992)). In both cases, an incentive scheme is introduced such that the regions are induced to follow a policy leading to a socially desirable steadystate equilibrium level of the pollution stocks. That is, the non-cooperative steady-state levels of the pollution stocks under the incentive mechanism equal the steady-state level of the pollution stocks under optimal management. However, the path under the incentive mechanism that determines the transition to the steady-state is not the same as the optimal management path. Conversely, the intertemporal pollution taxation scheme we propose induces the regions to follow a policy leading to emissions identical to the efficient emission levels not only in the long run but also along the transition.

The cooperative solution is obtained as the result of the joint optimization problem that for the two-region version reads:

$$
\max _{v_{1}, v_{2}} J^{c}\left(v_{1}, v_{2}, \boldsymbol{p}_{0}\right)=\sum_{i=1}^{2} \int_{0}^{+\infty} e^{-\rho t}\left(v_{i}\left(A_{i}-\frac{v_{i}}{2}\right)-\frac{\varphi_{i}}{2} p_{i}^{2}\right) \mathrm{d} t,
$$

subject to: (11).

Next proposition characterizes the cooperative solution of this cooperative differential game. Here and in the rest of the paper a superscript $c$ stands for cooperative.

Proposition 2. Assuming interior solutions, the following vector of optimal controls

$$
v_{l}^{c}\left(p_{1}, p_{2}\right)=A_{l}+\beta_{l} \frac{\partial W^{c}}{\partial p_{l}}\left(p_{1}, p_{2}\right)=A_{l}+\beta_{l}\left(b_{l}^{c}+a_{l}^{c} p_{l}+e^{c} p_{3-l}\right), \quad l=1,2,
$$

constitutes an optimal solution of the optimal control (35), where $W^{c}\left(p_{1}, p_{2}\right)$ denotes the value function, given by:

$$
W^{c}\left(p_{1}, p_{2}\right)=\frac{1}{2} a_{1}^{c} p_{1}^{2}+\frac{1}{2} a_{2}^{c} p_{2}^{2}+e^{c} p_{1} p_{2}+b_{1}^{c} p_{1}+b_{2}^{c} p_{2}+d^{c}
$$


Constants $a_{l}^{c}, b_{l}^{c}, d^{c}, e^{c}, l=1,2$ satisfy the following system of six Riccati equations:

$$
\begin{aligned}
& \left(a_{i}^{c}\right)^{2} \beta_{i}^{2}+2 e^{c} k_{j i}+\left(e^{c}\right)^{2} \beta_{2}^{2}-a_{i}^{c}\left(2 c_{i}-2 k_{i i}+\rho\right)-\varphi_{i}=0, \quad i, j=1,2, i \neq j \\
& A_{i} a_{i}^{c} \beta_{i}+A_{j} e^{c} \beta_{j}+b_{j}^{c}\left(e^{c} \beta_{j}^{j}+k_{j i}\right)-b_{i}^{c}\left(c_{i}-k_{i i}-\beta_{i}^{2} a_{i}^{c}+\rho\right)=0, \quad i, j=1,2, i \neq j \\
& \left(A_{i}+\beta_{i} b_{i}^{c}\right)^{2}+\left(A_{j}+\beta_{j} b_{j}^{c}\right)^{2}-2 \rho d^{c}=0, \quad i \neq j, \\
& e^{c}\left(c_{i}+c_{j}-k_{i i}-k_{j j}-a_{i}^{c} \beta_{i}^{2}-a_{j}^{c} \beta_{j}^{2}+\rho\right)-a_{i}^{c} k_{i j}-a_{j}^{c} k_{j i}=0, \quad i \neq j .
\end{aligned}
$$

Proof. The method is similar to that used in the proof of Proposition 1 . The only difference is that in this cooperative scenario there is a unique decisor agent and, therefore, a unique value function.

We first use Proposition 2 to characterize the cooperative solution for Example 1 which assumes a completely symmetric spatial relationship among regions $\Omega_{1}$ and $\Omega_{2}$ (a symmetric matrix $K$ ), allowing us to look for symmetric emission cooperative strategies and symmetric value function. Therefore, $a_{2}^{c}=a_{1}^{c}, \quad b_{2}^{c}=b_{1}^{c}$, and the system of six Ricatti equation (38)-(41) reduces to four equations with unknowns $a_{1}^{c}, b_{1}^{c}, e^{c}, d^{c}$. From (39), (40) and (41), respectively, the following expressions can be easily derived:

$$
b_{1}^{c}=\frac{A\left(a_{1}^{c}+e^{c}\right) \beta}{c-a^{c} \beta^{2}-e^{c} \beta^{2}+\rho}, \quad d^{c}=\frac{\left(A+b^{c} \beta\right)^{2}}{\rho}, \quad e^{c}=\frac{2 a_{1}^{c}}{2(1+c)-2 \beta^{2} a_{1}^{c}+\rho} .
$$

Substituting $e^{c}$ from (42) in (38), one gets a fourth-order polynomial equation in variable $a_{1}^{c}$ :

$$
\begin{gathered}
4 \beta^{6}\left(a_{1}^{c}\right)^{4}-8 \beta^{4}(2+2 c+\rho)\left(a_{1}^{c}\right)^{3}-\beta^{2}\left(4-5(2+2 c+\rho)^{2}+4 \beta^{2} \varphi\right)\left(a_{1}^{c}\right)^{2} \\
-\quad(2+2 c+\rho)\left((2 c+\rho)(2 c+\rho+4)-4 \beta^{2} \varphi\right) a_{1}^{c}-(2+2 c+\rho)^{2} \varphi=0 .
\end{gathered}
$$

The above equation can be analytically solved using Mathematica 11.0. For the parameter values in (29) only one of the four solutions implies two negative eigenvalues of the matrix associated to the closed-loop dynamical system obtained after substituting the optimal emission strategies. This solution is given by $a_{1}^{c}=-0.403752$, and correspondingly from $(42), b_{1}^{c}=-0.27339, d^{c}=5.13523, e^{c}=-0.211527$.

Therefore, the cooperative emission strategies read:

$$
\left[\begin{array}{l}
v_{1}^{c}\left(p_{1}, p_{2}\right) \\
v_{2}^{c}\left(p_{1}, p_{2}\right)
\end{array}\right]=L\left[\begin{array}{l}
p_{1} \\
p_{2}
\end{array}\right]+\left[\begin{array}{l}
0.22661 \\
0.22661
\end{array}\right] \text { where } L=\left[\begin{array}{cc}
-0.403752 & -0.211527 \\
-0.211527 & -0.403752
\end{array}\right]
$$

As in the non-cooperative scenario both cooperative strategies $v_{1}^{c}$ and $v_{2}^{c}$ depend negatively on both pollution stocks. Furthermore, these strategies satisfy the symmetry condition $v_{1}^{c}\left(p_{1}, p_{2}\right)=v_{2}^{c}\left(p_{2}, p_{1}\right)$. The identical steady-state values are $\boldsymbol{p}^{c S S}=\left[p_{1}^{c S S}, p_{2}^{c S S}\right]^{T}=[0.203187,0.203187]^{T}$. The time paths of the pollution stock along the equilibrium strategy are given by (31) where the steady-state values are $\boldsymbol{p}^{c S S}$ and the eigenvalues are $\lambda_{1}=-2.69223$ and $\lambda_{2}=-1.11528$. 
As expected the comparison of the non-cooperative and cooperative scenarios shows lower emission rates when the game is played cooperatively. This result applies not only in the long run, but for any value of the pollution stocks.

We move now to Example 2 and use Proposition 2 to characterize the cooperative solution for this example which introduces an asymmetric factor related to the geographical position of the regions.

In this scenario we have to deal with six Ricatti equations given by (38)-(41). Under assumption (23), from equation (41) one gets:

$$
e^{c}=\frac{a_{1}^{c}+a_{2}^{c}}{3+2 c-\beta^{2}\left(a_{1}^{c}+a_{2}^{c}\right)+\rho} .
$$

Substituting this last expression in (38) for the first player and (39) for the second player, the following two equations relating coefficients $a_{1}^{c}$ and $a_{2}^{c}$ are obtained:

$\left(a_{1}^{c}+a_{2}^{c}\right)^{2} \beta^{2}+2\left(a_{1}^{c}+a_{2}^{c}\right)\left(3+2 c-\beta^{2}\left(a_{1}^{c}+a_{2}^{c}\right)+\rho\right)+\left(3+2 c-\beta^{2}\left(a_{1}^{c}+a_{2}^{c}\right)+\rho\right)^{2}\left(\left(a_{2}^{c}\right)^{2} \beta^{2}-a_{2}^{c}(4+2 c+\rho)-\varphi\right)=0$, $\left(a_{1}^{c}+a_{2}^{c}\right)^{2} \beta^{2}+2\left(a_{1}^{c}+a_{2}^{c}\right)\left(3+2 c-\beta^{2}\left(a_{1}^{c}+a_{2}^{c}\right)+\rho\right)+\left(3+2 c-\beta^{2}\left(a_{1}^{c}+a_{2}^{c}\right)+\rho\right)^{2}\left(\left(a_{1}^{c}\right)^{2} \beta^{2}-a_{1}^{c}(2+2 c+\rho)-\varphi\right)=0$.

We numerically solve this system using the parameter values in (29). From the multiple solutions the unique associated with a globally stable steady-state is chosen: $a_{1}^{c}=-0.368586, a_{2}^{c}=-0.237321$. Hence, $e^{c}=-0.131265$. Once the values of $a_{1}^{c}, a_{2}^{c}$ and $e^{c}$ are known, from equations (39) for the first player and (39) for the second player, a system of two linear equations for coefficients $b_{1}^{c}$ and $b_{2}^{c}$ can be obtained. The solution is $b_{1}^{c}=-0.192159, b_{2}^{c}=-0.127844$. Finally, from equation $(40) d^{c}=11.6633$.

The cooperative emission strategies read:

$$
\left[\begin{array}{l}
v_{1}^{c}\left(p_{1}, p_{2}\right) \\
v_{2}^{c}\left(p_{1}, p_{2}\right)
\end{array}\right]=L\left[\begin{array}{l}
p_{1} \\
p_{2}
\end{array}\right]+\left[\begin{array}{l}
0.307841 \\
0.372156
\end{array}\right] \text { where } L=\left[\begin{array}{ll}
-0.368586 & -0.131265 \\
-0.131265 & -0.237321
\end{array}\right] \text {. }
$$

The steady-state values are $\boldsymbol{p}^{c S S}=\left[p_{1}^{c S S}, p_{2}^{c S S}\right]^{T}=[0.267410,0.220823]^{T}$, and the time paths of the pollution stock along the equilibrium strategy are given by

$$
\begin{array}{r}
p_{1}^{c}(t)=p_{1}^{c S S}+\left(0.447214\left(p_{2}(0)-p_{2}^{c S S}\right)+0.723607\left(p_{1}(0)-p_{1}^{c S S}\right)\right) e^{\lambda_{1} t} \\
-\left(0.447214\left(p_{2}(0)-p_{2}^{c S S}\right)-0.276393\left(p_{1}(0)-p_{1}^{c S S}\right)\right) e^{\lambda_{2} t} \\
p_{2}^{c}(t)=p_{2}^{c S S}+\left(0.276393\left(p_{2}(0)-p_{2}^{c S S}\right)+0.447214\left(p_{1}(0)-p_{1}^{c S S}\right)\right) e^{\lambda_{1} t} \\
+\left(0.723607\left(p_{2}(0)-p_{2}^{v S S}\right)-0.447214\left(p_{1}(0)-p_{1}^{v S S}\right)\right) e^{\lambda_{2} t}
\end{array}
$$

where $\lambda_{1}=-1.3317$ and $\lambda_{2}=-3.27423$ are the eigenvalues of the matrix $H=K-c I+L$.

As in the previous example the comparison of the non-cooperative and cooperative scenarios shows greater emission rates when the game is played non-cooperatively. 
As already mentioned at the beginning of this subsection among the different environmental policy instruments we propose an intertemporal pollution taxation scheme to sustain the cooperative Pareto efficient outcome. This scheme assumes perfect information and is in line with the proposal in Benchekroun \& Long (1998). We show that there exist time-independent taxes per unit of emission rate that depend on the current level of the pollution stocks and induce the regions to emit at the efficient emission levels.

First we focus on how the tax policy implemented by the environmental regulator alters the incentives of the regions to emit. Under the tax scheme the optimization problem for player $i$ in the two-player version changes to:

$$
\max _{v_{1}, v_{2}} J^{c}\left(v_{1}, v_{2}, \boldsymbol{p}_{0}\right)=\sum_{i=1}^{2} \int_{0}^{+\infty} e^{-\rho t}\left(v_{i}\left(A_{i}-\frac{v_{i}}{2}\right)-\frac{\varphi_{i}}{2} p_{i}^{2}-\tau_{i}\left(p_{i}, p_{j}\right) v_{i}\right) \mathrm{d} t,
$$

subject to: (11),

where $\tau_{i}\left(p_{i}, p_{j}\right)$ denotes the tax per unit of emission rate and the superscript $t$ stands for tax.

Restricting our attention to linear tax rules, i.e. rules of the form $\tau_{i}\left(p_{i}, p_{j}\right)=t_{i i} p_{i}+t_{i j} p_{j}+f_{i}, i, j=$ $1,2, i \neq j$, next proposition characterizes the feedback Nash equilibrium of the differential game under such tax scheme.

Proposition 3. Assuming interior solutions, and linear tax rules given by $\tau_{i}\left(p_{i}, p_{j}\right)=t_{i i} p_{i}+t_{i j} p_{j}+$ $f_{i}, i, j=1,2, i \neq j$ the following vector of strategies

$v_{l}^{t}\left(p_{1}, p_{2}\right)=A_{l}+\beta_{l} \frac{\partial W_{l}^{t}}{\partial p_{l}}\left(p_{1}, p_{2}\right)-\tau_{l}\left(p_{1}, p_{2}\right)=A_{l}+\beta_{l}\left(b_{l l}+a_{l l} p_{l}+e_{l} p_{3-l}\right)-t_{l l} p_{l}-t_{l 3-l} p_{3-l}-f_{l}, \quad l=1,2$,

constitutes a Markov-perfect Nash equilibrium of the pollution game (45), where $W_{i}^{t}\left(p_{1}, p_{2}\right)$ denotes region $i$ 's value function under the tax scheme given by:

$$
W_{i}^{t}\left(p_{i}, p_{j}\right)=\frac{1}{2} a_{i i}^{t} p_{i}^{2}+b_{i i}^{t} p_{i}+d_{i}^{t}+\frac{1}{2} a_{i j}^{t} p_{j}^{2}+b_{i j}^{t} p_{j}+e_{i}^{t} p_{i} p_{j}, \quad i, j=1,2, i \neq j .
$$

Constants $a_{i i}^{t}, b_{i i}^{t}, d_{i}^{t}, a_{i j}^{t}, b_{i j}^{t}, e_{i}^{t}, i, j=1,2, i \neq j$ satisfy the following system of twelve algebraic Riccati 
equations:

$$
\begin{aligned}
& \left(a_{i i}^{t}\right)^{2} \beta_{i}^{2}+t_{i i}^{2}+2 e_{i}^{t}\left(k_{j i}-t_{j i} \beta_{j}+\beta_{j}^{2} e_{j}^{t}\right)-a_{i i}^{t}\left(2 c_{i}-2 k_{i i}+2 t_{i i} \beta_{i}+\rho\right)-\varphi_{i}=0, \\
& \left(A_{i}-f_{i}\right)\left(a_{i i}^{t} \beta_{i}-t_{i i}\right)+e_{i}^{t} \beta_{j}\left(A_{j}-f_{j}+b_{j j}^{t} \beta_{j}\right)+b_{i j}^{t}\left(k_{j i}+\beta_{j}\left(e_{j}^{t} \beta_{j}-t_{j i}\right)\right) \\
& \quad-b_{i i}^{t}\left(c_{i}-k_{i i}-\beta_{i}\left(\beta_{i} a_{i i}^{t}-t_{i i}\right)+\rho\right)=0, \\
& \left(A_{i}-f_{i}+\beta_{i} b_{i i}^{t}\right)^{2}+2 \beta_{j} b_{i j}^{t}\left(A_{j}-f_{j}+\beta_{j} b_{j j}^{t}\right)-2 \rho d_{i}^{t}=0, \\
& 2 e_{i}^{t} k_{i j}+\left(\beta_{i} e_{i}^{t}-t_{i j}\right)^{2}-a_{i j}^{t}\left(2 c_{j}-2 k_{j j}-2 \beta_{j}\left(\beta_{j} a_{j j}^{t}-t_{j j}\right)+\rho\right)=0, \\
& \left(\beta_{i} e_{i}^{t}-t_{i j}\right)\left(A_{i}-f_{i}\right)+b_{i i}^{t}\left(k_{i j}+\beta_{i}\left(\beta_{i} e_{i}^{t}-t_{i j}\right)\right)+\beta_{j} a_{i j}^{t}\left(A_{j}-f_{j}+\beta_{j} b_{j j}^{t}\right) \\
& \quad-b_{i j}^{t}\left(c_{j}-k_{j j}-\beta_{j}\left(a_{j j}^{t} \beta_{j}-t_{j j}\right)+\rho\right)=0, \\
& e_{i}^{t}\left(c_{i}+c_{j}-k_{i i}-k_{j j}-\beta_{i}\left(a_{i i}^{t} \beta_{i}-t_{i i}\right)-\beta_{j}\left(a_{j j}^{t} \beta_{j}-t_{j j}\right)+\rho\right)-t_{i i} t_{i j}-a_{i i}^{t}\left(k_{i j}-t_{i j} \beta_{i}\right) \\
& \quad-a_{i j}^{t}\left(k_{j i}+\beta_{j}\left(e_{j}^{t} \beta_{j}-t_{j i}\right)\right)=0,
\end{aligned}
$$

$i, j=1,2, i \neq j$.

Proof. The method is similar to that used in the proof of Proposition 1.

Next proposition characterizes the optimal tax rule in each region.

Proposition 4. For the two-player case, the optimal tax that guides each region to achieve the efficient path as a Markov-perfect Nash equilibrium is:

$$
\tau_{i}\left(p_{i}, p_{j}\right)=t_{i i}^{*} p_{i}+t_{i j}^{*} p_{j}+f_{i}^{*}, \quad i, j=1,2, i \neq j
$$

where

$$
t_{i i}^{*}=\left(a_{i i}^{t}-a_{i}^{c}\right) \beta_{i}, t_{i j}^{*}=\left(e_{i}^{t}-e^{c}\right) \beta_{i}, f_{i}=\left(b_{i i}^{t}-b_{i}^{c}\right) \beta_{i}, \quad i, j=1,2, i \neq j .
$$

Proof. The result immediately comes from equating (46) to (36), that is, equating the feedback Nash equilibrium emissions of the differential game under the linear tax scheme to the optimal emissions of the cooperative scenario.

For Example 1, as in the non-cooperative scenario (before taxes) analyzed in Subsection 3.1.1, we look for symmetric strategies and consequently for symmetric linear tax rules, that is, $t_{j j}=t_{i i}, t_{j i}=$ $t_{i j}, f_{j}=f_{i}, i, j=1,2, i \neq j$. Taking into account the optimal emissions of the cooperative scenario given in (43) and the relationships in (54) the Riccati equations (48)-(53) can be manipulated following the same steps as in the non-cooperative scenario without taxes. The unique solution reads: $a_{i i}^{t}=$ $-0.316867, e_{i}^{t}=-0.074057, b_{i i}^{t}=-0.118902, a_{i j}^{t}=-0.027078, b_{i j}^{t}=-0.0903836, d_{i}^{t}=0.519421$. 
Therefore, the optimal tax that induces each region to achieve the efficient path reads:

$$
\left[\begin{array}{l}
\tau_{1}\left(p_{1}, p_{2}\right) \\
\tau_{2}\left(p_{1}, p_{2}\right)
\end{array}\right]=L\left[\begin{array}{l}
p_{1} \\
p_{2}
\end{array}\right]+\left[\begin{array}{l}
0.154488 \\
0.154488
\end{array}\right] \text { where } L=\left[\begin{array}{cc}
0.0868855 & 0.13747 \\
0.13747 & 0.0868855
\end{array}\right] .
$$

As expected each tax is positive related to both pollution stocks. The tax mechanism is defined in such a way that the external damages are completely internalized. Therefore, the tax rule should be able to induce both regions to internalize the environmental damage caused by its own pollution stock as well as by the pollution stock in the neighbouring region. As already commented, in the noncooperative scenario the marginal effect of an increase of the own pollution stock is greater (in absolute terms) than the marginal effect of an increase of the pollution stock accumulated in the other region (see (30)). That is, each region is able to autonomously internalize to a greater extent the environmental damages caused by its pollution stock than those caused by the pollution stock of the neighbouring region. Therefore, the corrective tax in each region should be more sensitive to any increase in the pollution stock accumulated in the neighbouring region to completely internalize the environmental damages.

For Example 2 the environmental regulator would need region specific (asymmetric) tax rules to achieve the first best. Taking into account the optimal emissions of the cooperative scenario given in (44) and the relationships in (54) the Riccati equations (48)-(53) can be manipulated following the same steps as in the non-cooperative scenario without taxes. The unique solution reads:

$$
\begin{aligned}
& a_{i i}^{t}=-0.327712, e_{i}^{t}=-0.0703723, b_{i i}^{t}=-0.129908, a_{i j}^{t}=-0.02252, b_{i j}^{t}=-0.0650445, d_{i}^{t}=2.31763 \\
& a_{j j}^{t}=-0.20325, e_{j}^{t}=-0.0429394, b_{j j}^{t}=-0.061897, a_{j i}^{t}=-0.01832, b_{j i}^{t}=-0.0619549, d_{j}^{t}=5.01778
\end{aligned}
$$

Therefore, the optimal tax that induces each region to achieve the efficient path read:

$$
\left[\begin{array}{l}
\tau_{1}\left(p_{1}, p_{2}\right) \\
\tau_{2}\left(p_{1}, p_{2}\right)
\end{array}\right]=L\left[\begin{array}{l}
p_{1} \\
p_{2}
\end{array}\right]+\left[\begin{array}{c}
0.0622505 \\
0.065947
\end{array}\right] \text { where } L=\left[\begin{array}{ll}
0.0408737 & 0.0608927 \\
0.0883256 & 0.0340706
\end{array}\right] .
$$

These taxes would induce both regions to emit at the Pareto efficient level as a Markov-perfect Nash equilibrium. That is, the tax rule is designed such that the socially optimum time path of the pollution stocks are decentralized as a feedback Nash equilibrium of the dynamic game played by the two regions.

After the exhaustive study of the case of two players, we revisit now two of the examples analyzed in Section 3.2, specifically Examples 3 and 5, with 3 and 4 players, respectively.

For Example 3, Figure 9 presents the results of the comparison of the emission (left) and pollution stock (right) time-paths along the cooperative and non-cooperative strategies. In the figure, the superscript $C$ stands for 'cooperative'. 



Figure 9: Example 3. Emission (left) and pollution stock (right) time-paths along the non-cooperative and cooperative strategies

As expected in a cooperative framework the three regions reduce the optimal emission levels at each time with respect to these levels in the non-cooperative scenario. Let us recall that in this example region 2 shares boundary with regions 1 and 3, while the last two (identical) regions share boundary with region 2 and with the exterior of the whole region $\Omega$. Under cooperation, region 2 , taking advantage of its geographical position, still emits over the other two regions which emit at an identical rate. However, as Figure 9 shows the difference between the emission levels is substantially greater under noncooperation than under cooperation. The effect of the reduction of the emission levels in the cooperative scenario unequivocally leads to lower pollution stocks time-paths along the whole time horizon. In the cooperative scenario the pollution stocks of the three regions are almost identical along the optimal trajectory. The environmental regulator (the unique decision maker in the cooperative setting) chooses the emissions in the three regions in such a way that all regions suffer a similar environmental damage because of an almost identical time path of the pollution stock along the cooperative solution.

For Example 5 and for ease of presentation we present on the left side of Figures 10 and 11 the emission and pollution stock time-paths along the non-cooperative equilibrium strategies; and on the right side of these figures the time-paths along the cooperative solution. Figure 10 shows that as in the previous example, region 4 takes advantage of its geographical position (region 4 shares a boundary with the exterior of the whole region $\Omega$ which is assumed to have a zero concentration of pollutants, $\left.p_{40}=0\right)$. Along the cooperative solution and at each point in time region 4 emits more than any other region. In the cooperative framework, the environmental regulator optimally chooses a smoother behaviour for the optimal time-paths of the emission level of the different regions. However, unlike the 

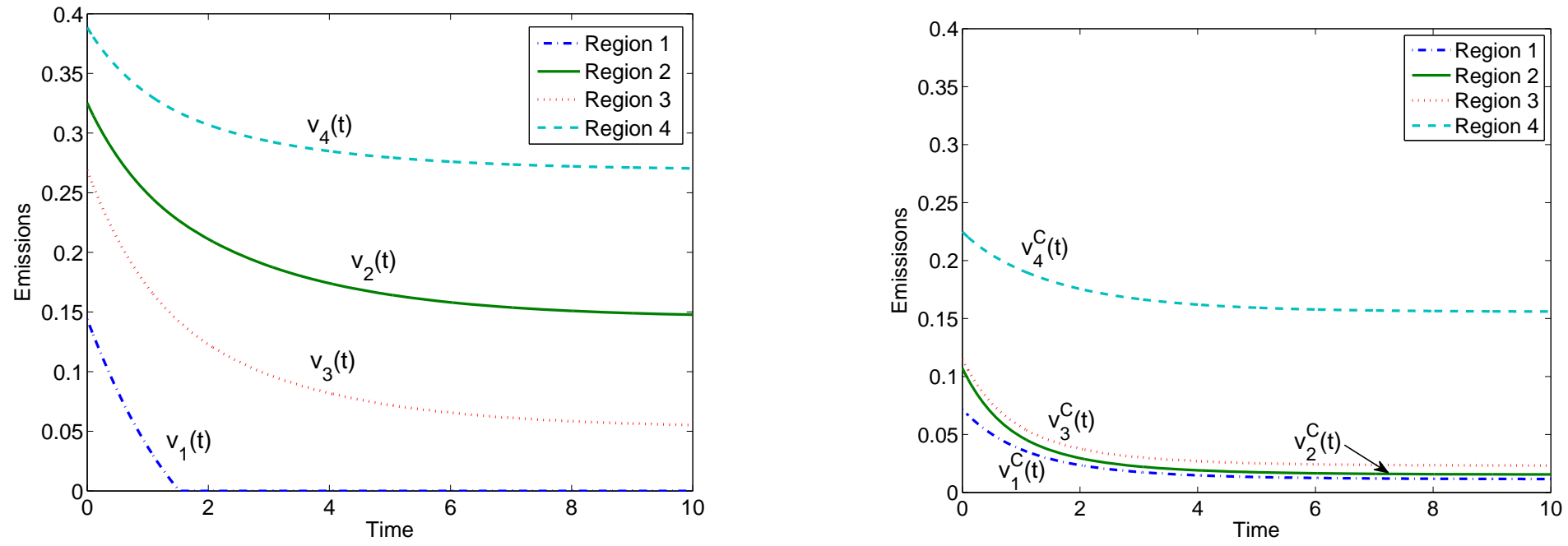

Figure 10: Example 5. Emission time-paths along the non-cooperative (left) and cooperative (right) strategies
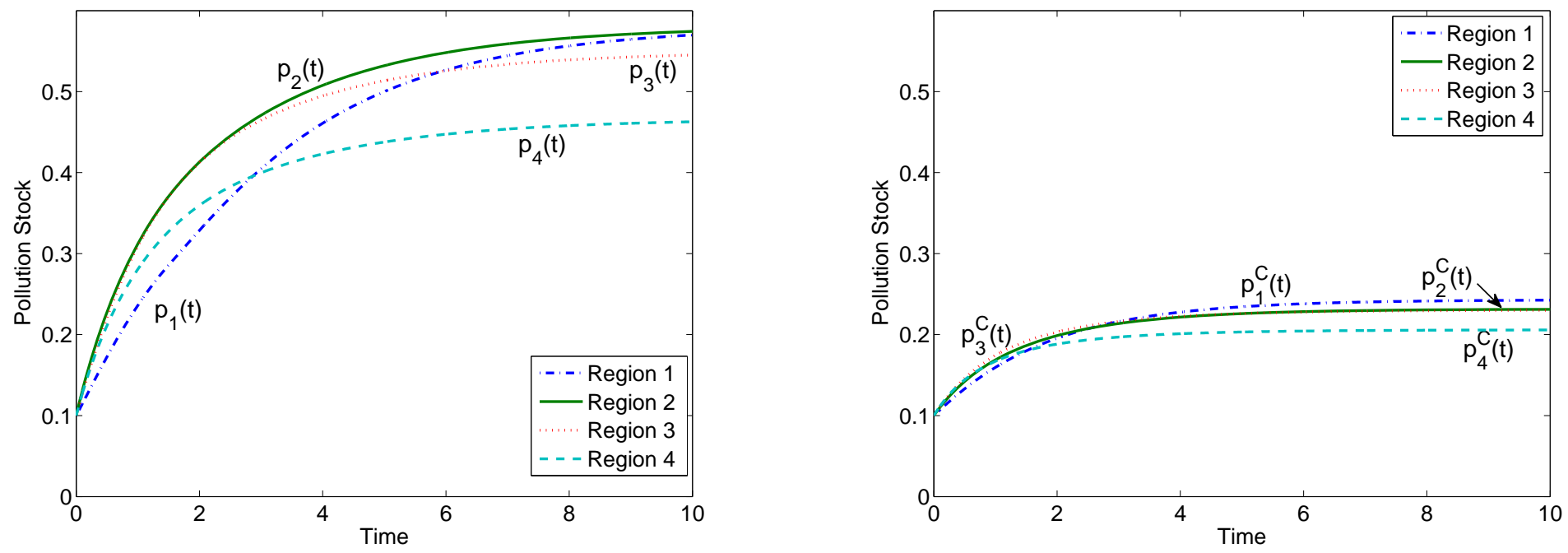

Figure 11: Example 5. Pollution stock time-paths along the non-cooperative (left) and cooperative (right) strategies

previous example, one of the regions (region 1) increases its optimal emission level with respect to its non-cooperative level. In the non-cooperative framework region 1 in a first period of time emits at a much lower level than the other regions, and after this initial period it does not emit at all. Importantly, region 1 in the cooperative setting emits along the whole time horizon at a positive rate. The emission time-paths along the cooperative solution are very similar for regions 1, 2 and 3 , although they were much different in the non-cooperative case.

Finally, Figure 11 illustrates that the regulator's choice of more uniform emission levels between 
regions leads to pollution time-paths along the cooperative solution much lower than the corresponding non-cooperative levels. These cooperative emission levels are very similar for the four regions, both in the short and in the long run.

\section{Concluding remarks}

This paper analyzes a transboundary pollution dynamic game with spatial effects and strategic decision makers. The paper tries to make a contribution to the quite recent literature that has added the spatial dimension to the standard (temporal) dynamic models used to study economic and environmental problems. The technical difficulties that arise when optimization takes place in spatio-temporal domains are undoubtedly the reason for the lack of abundant literature on this subject. However, recently some authors have introduced in different economic contexts this spatial dimension. As revised in the introduction, these contributions have focussed either on the problem of a social planner or on special private optimization problems. In the first case, the social planner allocates resources to maximize the present value of an objective over the entire spatial domain subject to the spatio-temporal evolution of the state variable(s). In the second case, a special structure is added to the problem in such a way that the economic agents behave myopically in both the temporal and the spatial dimensions, and therefore, agents solve static problems. Departing from these two settings, this paper focussed on the analysis of a transboundary pollution problem with spatial diffusion in a multiregional setting, considering agents who behave dynamically and strategically. We present a first characterization of the equilibrium outcomes of an intertemporal pollution problem where there is a continuum of spatial sites and the pollution stock diffuses over these sites.

Originally, we state the model in continuous space and continuous time with two spatial dimensions and one temporal dimension. The planar region of interest is divided in $J$ subregions. In each subregion there is a decision-maker who decides the emission level in order to maximize the present value of benefits net of environmental damages due to the concentration of pollutants over his subregion. The dynamic optimization problem is subject to the spatio-temporal evolution of the stock of a pollutant, described by a Diffusion PDE. An essential difference with respect to the previous literature is that each economic agent responsible for controlling the emissions at each subregion when deciding the optimal emission in order to maximize his profits takes into account the spatial transport phenomena across space (across all the planar region of interest). Therefore, our original formulation is a $J$-player differential game with one control variable for each player (the emission decision at his subregion) and one infinite-dimensional state variable (the stock of a pollutant). The Markovian Nash Equilibrium of this differential game 
is approximated by the solution of a simplified model which still captures the spatial interactions. This simplified model can be interpreted as a spatial discretization of the original model. This spacediscretized model has $J$ players and $J$ state variables, each one describing the average pollution in one subregion. Each player decides the average total emission in his subregion in order to maximize his profits net of environmental damages subject to the time evolution of $J$ state variables described by a system of $J$ ordinary differential equations (ODEs).

For the two-player specification we analytically characterized the feedback Nash equilibrium of the space-discretized model. The solutions of this model with the number of players is greater than two are characterized using a numerical algorithm that solves an approximate time-discrete dynamic game. Our numerical examples show, on the one hand, how the equilibrium emission policies in a spatial context differ from those characterized ignoring the spatial dimension. On the other hand, the comparison of the equilibrium emission policies we have obtained in our differential game version and those obtained for the same model when the decision maker is an environmental regulator allows us to evaluate the impact of the strategic and spatially dynamic behaviour of the agents on the design of equilibrium environmental policies. An intertemporal pollution taxation scheme to sustain the cooperative Pareto efficient outcome is proposed.

One of the possible extensions that we think merit exploration in more detail include the addition of a second dimension for the pollution. In the present formulation pollution has a local dimension as a direct consequence of the production of the consumption good in a particular region. However, additionally pollution produced in other regions may also harm welfare. In this case, the environmental damage function would depend on the pollution over the entire spatial domain. The double dimension (local and global) of pollution in a different framework has been already introduced in Camacho \& PérezBarahona (2015) in their study of optimal land use and environmental degradation. This analysis is one of the subjects of our future research.

\section{Appendix A: Technical hypotheses and definitions}

The spatio-temporal dynamics of the stock of pollution is given by the following parabolic partial differential equation:

$$
\begin{aligned}
& \frac{\partial P}{\partial t}=\nabla \cdot(k \nabla P)+\boldsymbol{b}^{T} \nabla P-c P+N(P)+F(\boldsymbol{u}), \boldsymbol{x} \in \Omega, \\
& P(\boldsymbol{x}, 0)=P_{0}(\boldsymbol{x}), \quad \boldsymbol{x} \in \Omega, \\
& \alpha(\boldsymbol{x}) P(\boldsymbol{x}, t)+k(\boldsymbol{x}) \nabla P^{T}(\boldsymbol{x}, t) \boldsymbol{n}=\alpha(\boldsymbol{x}) P_{b}(\boldsymbol{x}, t), \quad \boldsymbol{x} \in \partial \Omega .
\end{aligned}
$$


Here $\boldsymbol{u}=\left[u_{1}, \ldots, u_{J}\right]^{T}$ is the vector of emission rates, $k=k(\boldsymbol{x})$ is a local diffusion coefficient which is assumed to be a smooth function satisfying $k_{m} \leq k(\boldsymbol{x}) \leq k_{M}$, for all $\boldsymbol{x} \in \Omega$, where $0<k_{m} \leq k_{M}$ are two given constants. The coefficient $k=k(\boldsymbol{x})$ is a characteristic diffusion parameter that can depend on $\boldsymbol{x} \in \Omega$. It measures the velocity at which the stock of pollution is diffused away in a particular location $\boldsymbol{x}$. The vector $\boldsymbol{b}=\boldsymbol{b}(\boldsymbol{x}, t)$ is a smooth convective field which can be time dependent. The term $\boldsymbol{b}^{T} \nabla P$ accounts for a transport phenomenon. Pollution is transported in the direction of the vector field $\boldsymbol{b}$ with speed $\left(\boldsymbol{b}^{T} \boldsymbol{b}\right)^{1 / 2}$. We assume, for simplicity, that $\nabla \cdot \boldsymbol{b}=0$. The term $N(P)$ denotes a possibly non-linear reaction function and $c P, c=c(\boldsymbol{x}, t) \geq 0$, is a natural decay of the pollutant.

Along the paper we assume the following set of technical hypotheses some of which have been already stated in the body of the manuscript and that are included here for the reader's convenience.

H1 The regions $\Omega \subset \mathbb{R}^{2}$ and $\Omega_{i}, i=1, \ldots, J$ defined in (1), are bounded Lipschitz domains.

H2 The diffusion coefficient in $(57), k(\boldsymbol{x})$, is continuously differentiable in $\Omega$. There exist two constants $0<k_{m} \leq k_{M}$ such that $k_{m}<k(\boldsymbol{x})<k_{M}$, for all $\boldsymbol{x} \in \Omega$. The velocity field $\boldsymbol{b}(\boldsymbol{x})$ is continuously differentiable. Furthermore, $\nabla \cdot \boldsymbol{b}=0$. Function $c$ is continuously differentiable and non negative.

H3 The non-linear function $N(P)$ in $(57)$ is continuously differentiable and either monotone decreasing or globally Lipschitz continuous.

H4 Function $F$ in (57) is of the form (4) with $F_{i}$ monotone increasing differentiable functions with Lipschitz derivative defined for $u_{i} \in \mathbb{U}_{i}$, where $\mathbb{U}_{i} \subset \mathbb{R}_{+}$is closed and bounded.

H5 The functions $B_{i}, i=1, \ldots, J$, in (6) are two times differentiable, concave, non-decreasing functions defined in $\mathbb{U}_{i}$. The functions $D_{i}, i=1, \ldots, J$, in (6) are two times differentiable convex functions defined in $\mathbb{R}$.

H6 The initial pollution state $P_{0}(\boldsymbol{x})$ is a function in $L^{2}(\Omega)$, the space of square integrable functions defined in $\Omega$.

For simplicity in the notation we will write $v(t)$ to represent a generic function $v=v(\boldsymbol{x}, t)$, such that $v(\cdot, t) \in H$ where $H$ is a given function space. Let $\mathbb{U}_{i}$ the set of functions $v \in L^{2}\left(\Omega_{i}\right)$ such that $v(\boldsymbol{x}) \in \mathbb{U}_{i}$ for almost every $\boldsymbol{x} \in \Omega_{i}$. We define the set of admissible controls $\mathcal{U}_{i}$ as the set of functions $u_{i}:[0, T] \rightarrow U_{i}$ with $u_{i} \in L^{2}\left(\Omega_{i} \times(0, T)\right)$.

The set of hypotheses above guarantees that for each choice of controls $u_{i}(\boldsymbol{x}, t)$, with $u_{i} \in \mathcal{U}_{i}$ the state equation (57) has a unique (weak) solution $P \in \mathcal{C}\left([0, T] ; L^{2}(\Omega)\right) \cap L^{2}\left((0, T) ; H^{1}(\Omega)\right)$, for all $T>0$, (see, for example, Barbu (1993), Tröltzsch (2009), Liu \& Yong (1995)). Here, and in the rest of the paper, $H^{r}(\Omega)$ represents the Sobolev space of functions with $r$ distributional derivatives in $L^{2}(\Omega)$. 
We are interested in finding stationary Markov-perfect Nash equilibria of the dynamic game defined by (57), (4)-(6) so that we look for controls $u_{i} \in \mathcal{U}_{i}$ of the form $u_{i}(t)=\Lambda_{i}(P(t))$, where the strategies $\Lambda_{i}: L^{2}(\Omega) \rightarrow U_{i}$, are assumed to be compatible with the dynamics (57) in the sense that the closed-loop system

$$
\begin{aligned}
& \frac{\partial P}{\partial t}=\nabla \cdot(k \nabla P)+b^{T} \nabla P-c P+N(P)+F(\boldsymbol{\Lambda}(P(t), t)), \boldsymbol{x} \in \Omega, \\
& P(\boldsymbol{x}, \tau)=P_{\tau}(\boldsymbol{x}), \quad \boldsymbol{x} \in \Omega, \\
& \alpha P(\boldsymbol{x}, t)+k(\boldsymbol{x}) \nabla P^{T}(\boldsymbol{x}, t) \boldsymbol{n}=\alpha P_{b}(\boldsymbol{x}, t), \quad \boldsymbol{x} \in \partial \Omega,
\end{aligned}
$$

has a unique solution defined in $[\tau, \infty)$ for every $\tau \geq 0$ and every initial condition $P_{\tau}$. Here, we are using the notation $\boldsymbol{\Lambda}=\left[\Lambda_{1}, \ldots, \Lambda_{J}\right]^{T}$.

A $J$-tuple of admissible strategies $\Lambda^{*}=\left[\Lambda_{1}^{*}, \ldots, \Lambda_{J}^{*}\right]^{T}$ are a Markov-perfect Nash equilibrium if for all initial state $P_{0}$,

$$
J_{i}\left(\boldsymbol{u}^{*}, P_{0}\right) \geq J_{i}\left(\left[u_{i}, \boldsymbol{u}_{-i}^{*}\right], P_{0}\right), \quad \forall u_{i} \in \mathcal{U}_{i}
$$

where $\boldsymbol{u}^{*}=\left[u_{1}^{*}, \ldots, u_{J}^{*}\right]^{T}, u_{j}^{*}(t)=\Lambda_{j}^{*}\left(P^{*}(t)\right), P^{*}$ is the solution of (58) with $\boldsymbol{\Lambda}=\boldsymbol{\Lambda}^{*}$ and $\left[u_{i}, \boldsymbol{u}_{-i}^{*}\right]=$ $\left[u_{1}^{*}, \ldots, u_{i}, \ldots, u_{J}^{*}\right]$. Here, for simplicity, we are using the concept of strong optimality. When dealing with the infinite horizon problem other possibilities are also advisable, see Haurie et al. (2012).

Let us suppose that $\Lambda_{i}^{*}(P), i=1 \ldots, J$, are a stationary Markov-perfect Nash equilibrium. Then the value functions $V^{i}, i=1, \ldots, J$, satisfy, for $P \in H^{2}(\Omega)$, the infinite-dimensional Hamilton-JacobiBellman system

$$
\rho V^{i}(P)=\sup _{u_{i} \in U_{i}}\left\{\mathcal{G}^{i}\left(P,\left[u_{i}, \Lambda_{-i}^{*}\right]\right)+\left\langle\mathcal{F}\left(P,\left[u_{i}, \Lambda_{-i}^{*}\right]\right), \nabla V^{i}\right\rangle\right\}
$$

with transversality condition

$$
\lim _{T \rightarrow \infty} e^{-\rho T} V^{i}(P(T))=0
$$

Here, we are using the notations

$$
\mathcal{G}^{i}\left(P, u_{1}, \ldots, u_{J}\right)=\int_{\Omega_{i}} G_{i}\left(P, u_{1}, \ldots, u_{J}\right) d \boldsymbol{x}
$$

and

$$
\mathcal{F}\left(P, u_{1}, \ldots, u_{J}\right)=\nabla \cdot(k \nabla P)+\boldsymbol{b} \cdot \nabla P-c P+N(P)+F\left(u_{1}, \ldots, u_{J}\right),
$$

the brackets represent the $L^{2}(\Omega)$ inner product and $\nabla V^{i}$ denotes the Fréchet derivative of the function $V^{i}$ with respect to $P$.

Furthermore, $\Lambda_{i}^{*}(P)$ is a maximizer of the right hand side of $(59)$ and $V^{i}\left(P_{0}\right)=J_{i}\left(\boldsymbol{u}^{*}, P_{0}\right)$. We refer to Başar \& Olsder (1999), Haurie et al. (2012), for a proof of this result in the finite dimensional case. See also Li \& Yong (1995). 
The Hamilton-Jacobi-Bellman system (59) is a non-linear infinite-dimensional system. It is well known that even in the finite dimensional the solutions of (59) can fail to have enough regularity and one has to resort to generalized solutions. Let us remark that, even in the finite dimensional case, problem (59) with the transversality condition (60) can have multiple solutions. We refer to Cannarsa \& Da Prato (1990), Barbu (1993), Li \& Yong (1995) for a deep analysis in the case $J=1$. The analysis of (59) is out of the scope of this paper and it will be the subject of further research.

\section{Appendix B: The discrete-space model}

We present in this appendix the details of the derivation of the discrete-space model, which can be seen as a space discretization of the continuous space model state in Section 2.

We will use aggregated variables. Let us denote by $m_{i}=\int_{\Omega_{i}} \mathrm{~d} \boldsymbol{x}$ the area of region $\Omega_{i}, i=1, \ldots, J$. We consider the averaged stock of pollution over region $\Omega_{i}$,

$$
p_{i}(t)=\frac{1}{m_{i}} \int_{\Omega_{i}} P(\boldsymbol{x}, t) \mathrm{d} \boldsymbol{x}, \quad i=1, \ldots, J
$$

and the averaged emissions over $\Omega_{i}$

$$
v_{i}(t)=\frac{1}{m_{i}} \int_{\Omega_{i}} u_{i}(\boldsymbol{x}, t) \mathrm{d} \boldsymbol{x}, \quad i=1, \ldots, J
$$

Integrating equation (57) over region $\Omega_{i}, i=1, \ldots, J$ and using (4) we have

$$
\int_{\Omega_{i}} \frac{\partial P}{\partial t} \mathrm{~d} \boldsymbol{x}=\int_{\Omega_{i}}\left(\nabla \cdot(k \nabla P)+\boldsymbol{b}^{T} \nabla P-c P+N(P)+F_{i}\left(u_{i}\right)\right) \mathrm{d} \boldsymbol{x} .
$$

In order to simultaneously treat the cases $\partial \Omega_{i} \cap \partial \Omega=\emptyset$ (interior subdomains) and $\partial \Omega_{i} \cap \partial \Omega \neq \emptyset$ (boundary subdomains with a part of their boundary over $\partial \Omega$ ), we introduce a fictitious subdomain $\Omega_{0}=\mathbb{R}^{2} \backslash \bar{\Omega}$. In this way $\partial_{i, 0}=\bar{\Omega}_{i} \cap \bar{\Omega}_{0}=\partial \Omega_{i} \cap \partial \Omega$.

We use the divergence theorem in the first term on the right to arrive to

$$
\int_{\Omega_{i}} \nabla \cdot(k \nabla P) \mathrm{d} \boldsymbol{x}=\int_{\partial \Omega_{i}} k \nabla P^{T} \boldsymbol{n}_{i} \mathrm{~d} \boldsymbol{\sigma}(\boldsymbol{x})=\sum_{\substack{j=0 \\ \partial_{i j} \neq \emptyset}}^{J} \int_{\partial_{i j}} k \nabla P^{T} \boldsymbol{n}_{i} \mathrm{~d} \boldsymbol{\sigma}(\boldsymbol{x}) \approx \sum_{\substack{j=0 \\ \partial_{i j} \neq \emptyset}}^{J} k_{i j}\left(p_{j}-p_{i}\right),
$$

where $\boldsymbol{n}_{i}$ is the normal vector pointing outwards $\Omega_{i}$, and

$$
k_{i j}=\frac{L_{i j}}{\operatorname{length}\left(\partial_{i j}\right)} \int_{\partial_{i j}} k \mathrm{~d} \boldsymbol{\sigma}(\boldsymbol{x}), \quad j=1, \ldots, J .
$$


Here $L_{i j}$ is a scaling parameter proportional to the ratio between length $\left(\partial_{i j}\right)$ and the distance between the center of masses of the subdomains $\Omega_{i}$ and $\Omega_{j}$. In the case of a boundary subdomain, $j=0$, the term $\int_{\partial_{i j}} k \nabla P^{T} \boldsymbol{n}_{i} \mathrm{~d} \boldsymbol{\sigma}(\boldsymbol{x})$ is computed using the boundary condition in (57), so that we approximate

$$
\int_{\partial_{i 0}} k \nabla P^{T} \boldsymbol{n}_{i} \mathrm{~d} \boldsymbol{\sigma}(\boldsymbol{x}) \approx \alpha \operatorname{length}\left(\partial_{i 0}\right)\left(p_{i 0}-p_{i}\right), \quad \text { with } \quad p_{i 0}=\frac{1}{\operatorname{length}\left(\partial_{i 0}\right)} \int_{\partial_{i 0}} P_{b}(\boldsymbol{x}) \mathrm{d} \boldsymbol{\sigma}(\boldsymbol{x}) .
$$

For simplicity in the presentation, we assume that $p_{i 0}=p_{0}$, for all $i=1, \ldots, J$, and write $k_{i 0}=$ $\alpha \operatorname{length}\left(\partial_{i 0}\right)$.

The second term is treated similarly:

$$
\int_{\Omega_{i}} \boldsymbol{b}^{T} \nabla P \mathrm{~d} \boldsymbol{x}=\int_{\partial \Omega_{i}} P\left(\boldsymbol{b}^{\boldsymbol{T}} \boldsymbol{n}_{\boldsymbol{i}}\right) \mathrm{d} \boldsymbol{x}=\sum_{\substack{j=0 \\ \partial_{i j} \neq \emptyset}}^{J} \int_{\partial_{i j}} P\left(\boldsymbol{b}^{\boldsymbol{T}} \boldsymbol{n}_{\boldsymbol{i}}\right) \mathrm{d} \boldsymbol{\sigma}(\boldsymbol{x}) \approx \sum_{\substack{j=0 \\ \partial_{i j} \neq \emptyset}}^{J} b_{i j} \varphi\left(p_{i}, p_{j}\right),
$$

with

$$
b_{i j}=\int_{\partial_{i j}}\left(\boldsymbol{b}^{\boldsymbol{T}} \boldsymbol{n}_{\boldsymbol{i}}\right) \mathrm{d} \boldsymbol{\sigma}(\boldsymbol{x}) .
$$

Here we have used the condition $\nabla \cdot \boldsymbol{b}=0$. There are several possible choices for the numerical flux $\varphi\left(p_{i}, p_{j}\right)$. The choice $\varphi\left(p_{i}, p_{j}\right)=\left(p_{i}+p_{j}\right) / 2$ gives a term that is reminiscent of second-order centered finite difference approximations. This choice is reasonable in problems in which diffusion is dominant, that is, if $\max k(x)$ is large compared with $\max |\boldsymbol{b}(x)|$. An alternative is to use an upwind flux defined, in the simplest case, by $\varphi\left(p_{i}, p_{j}\right)=p_{i}$ if $b_{i j} \leq 0$ and $\varphi\left(p_{i}, p_{j}\right)=p_{j}$ if $b_{i j}>0$. With this choice one can deal with convection dominated problems.

The rest of the terms are handle as follows

$$
\begin{gathered}
\int_{\Omega_{i}} c P \mathrm{~d} \boldsymbol{x} \approx c_{i} p_{i}, \quad \text { with } \quad c_{i}=\int_{\Omega_{i}} c(\boldsymbol{x}) \mathrm{d} \boldsymbol{x} \\
\int_{\Omega_{i}} N(P) \mathrm{d} \boldsymbol{x} \approx m_{i} N\left(p_{i}\right)
\end{gathered}
$$

and

$$
\int_{\Omega_{i}} F_{i}\left(u_{i}\right) \mathrm{d} \boldsymbol{x} \approx m_{i} F_{i}\left(v_{i}\right) .
$$

The term with the partial derivative with respect time is approximated also using the aggregated stock $p_{i}(t)$ by

$$
\int_{\Omega_{i}} \frac{\partial P}{\partial t} \mathrm{~d} \boldsymbol{x} \approx m_{i} \frac{\mathrm{d} p_{i}}{\mathrm{~d} t} .
$$

All in all, the dynamics of the aggregated stock of pollution is

$$
m_{i} \frac{\mathrm{d} p_{i}}{\mathrm{~d} t}=\sum_{\substack{j=0 \\ j \neq i}}^{J} k_{i j}\left(p_{j}-p_{i}\right)+\sum_{\substack{j=0 \\ j \neq i}}^{J} b_{i j} \varphi\left(p_{i}, p_{j}\right)-m_{i} c_{i} p_{i}+m_{i} N\left(p_{i}\right)+m_{i} F_{i}\left(v_{i}\right), \quad i=1, \ldots, J .
$$


We note that (64) is a system of ordinary differential equations. The system is supplemented with the initial conditions given by

$$
p_{i}(0)=\frac{1}{m_{i}} \int_{\Omega_{i}} P_{0}(\boldsymbol{x}) \mathrm{d} \boldsymbol{x}:=p_{i}^{0}, \quad i=1, \ldots, J,
$$

where $P_{0}(\boldsymbol{x})$ is the initial data in (57).

The objective of player $i$ is approximated using that

$$
\int_{\Omega_{i}}\left(B_{i}\left(u_{i}\right)-D_{i}(P)\right) \mathrm{d} \boldsymbol{x} \approx m_{i}\left(B_{i}\left(v_{i}\right)-D_{i}\left(p_{i}\right)\right):=\widetilde{G}_{i}\left(v_{i}, p_{i}\right) .
$$

We state, finally, the discrete-space model: The objective of player $i$ is to maximize the space averaged payoff

$$
\widetilde{J}_{i}\left(v_{1}, \ldots, v_{J}, \boldsymbol{p}^{0}\right)=\int_{0}^{\infty} e^{-\rho t} \widetilde{G}_{i}\left(v_{i}, p_{i}\right) \mathrm{d} t
$$

subject to (64), where $\boldsymbol{p}^{0}=\left[p_{1}^{0}, \ldots, p_{J}^{0}\right]^{T}$.

\section{Appendix C: The numerical method}

As it is well known there is little hope to find analytical solutions to the problem at hand, so we approximate the solution by a numerical method adapted from De Frutos \& Martín-Herrán (2015) consisting mainly of two steps. In the first step we consider a time-discrete version of problem (64)(66). In the second step we discretize the state space.

Let $h>0$ a positive parameter and let $t_{n}=n h$, the discrete times defined for all positive integers $n$. We denote by $\delta_{h}=1-\rho h$ the discrete discount factor. In what follows $\bar{u}_{i}, i=1, \ldots, J$ denotes a sequence of real numbers $\bar{u}_{i}=\left\{u_{i, n}\right\}_{n=0}^{\infty}$ and $\mathcal{U}$ denotes the set of real sequences $\bar{v}$ with $v_{n} \geq 0$ for all $n \in \mathbb{N}$.

We introduce the notation $\boldsymbol{g}(\boldsymbol{p}, \boldsymbol{u})=\left[g_{1}(\boldsymbol{p}, \boldsymbol{u}), \ldots, g_{J}(\boldsymbol{p}, \boldsymbol{u})\right]^{T}$ to denote the function collecting the right hand side of (64). More precisely, for $\boldsymbol{p}=\left[p_{1}, \ldots, p_{J}\right]^{T} \in \mathbb{R}^{J}$ and $\boldsymbol{u}=\left[u_{1}, \ldots, u_{J}\right]^{T} \in \mathbb{R}^{J}$ with $u_{i} \geq 0, i=1, \ldots, J$,

$$
g_{i}(\boldsymbol{p}, \boldsymbol{u})=\sum_{\substack{j=0 \\ j \neq i}}^{J} \frac{k_{i j}}{m_{i}}\left(p_{j}-p_{i}\right)+\sum_{\substack{j=0 \\ j \neq i}}^{J} \frac{b_{i j}}{m_{i}} \varphi\left(p_{i}, p_{j}\right)-c_{i} p_{i}+N\left(p_{i}\right)+F_{i}\left(u_{i}\right) .
$$

We consider the time-discrete infinite horizon game in which player $i=1, \ldots, J$ aims to maximize

$$
W_{i}\left(\bar{u}_{i}, \boldsymbol{p}_{0}\right)=h \sum_{n=1}^{\infty} \delta_{h}^{n} \widetilde{G}_{i}\left(u_{i, n}, p_{i, n}\right), \quad \bar{u}_{i} \in \mathcal{U},
$$


subject to

$$
\boldsymbol{p}_{n+1}=\boldsymbol{p}_{n}+h \boldsymbol{g}\left(\boldsymbol{p}_{n}, \boldsymbol{u}_{n}\right), \quad n \geq 0,
$$

where $\boldsymbol{p}_{n}=\left[p_{1, n}, \ldots, p_{J, n}\right]^{T}, \boldsymbol{u}_{n}=\left[u_{1, n}, \ldots, u_{J, n}\right]^{T}$ and $\boldsymbol{p}_{0}$ is a given initial state.

It is worth noting that the time-discrete problem (67)-(68) can be seen as a discretization of the functional (66) by means of the rectangle rule followed by a forward Euler discretization of the dynamics in (64).

The time-discrete value function $V_{h, i}(\boldsymbol{p}), i=1, \ldots, J$, is computed solving the Bellman equations

$$
V_{h, i}(\boldsymbol{p})=\max _{u_{i} \geq 0}\left\{h \widetilde{G}_{i}\left(p_{i}, u_{i}\right)+\delta_{h} V_{h, i}\left(\boldsymbol{p}+h \boldsymbol{g}\left(\boldsymbol{p},\left[u_{i}, \boldsymbol{u}_{-i}^{*}\right]\right)\right)\right\},
$$

where, for $i=1, \ldots, J$,

$$
u_{i}^{*}=\arg \max _{u_{i} \geq 0}\left\{h \widetilde{G}_{i}\left(p_{i}, u_{i}\right)+\delta_{h} V_{h, i}\left(\boldsymbol{p}+h \boldsymbol{g}\left(\boldsymbol{p},\left[u_{i}, \boldsymbol{u}_{-i}^{*}\right]\right)\right)\right\} .
$$

Here, and in the rest of this section, we are using the notation

$$
\left[u_{i}, \boldsymbol{v}_{-i}\right]=\left[v_{1}, \ldots, v_{i-1}, u_{i}, v_{i+1}, \ldots, v_{J}\right]^{T}, \quad u_{i} \in \mathbb{R}, \boldsymbol{v} \in \mathbb{R}^{J}
$$

The solution of system (69) is approximated using a collocation method based on tensorial product of linear splines. Of course other type of discretizations are advisable. In De Frutos \& Martín-Herrán (2015) a state discretization based on monotone cubic splines has been used. In Hager (2000) RungeKutta time discretizations for optimal control problems have been analyzed.

Let us introduce a positive parameter $P_{M}>0$ big enough and consider, for a given integer $N>0$ a partition of the interval $\left[0, P_{M}\right] \subset \mathbb{R}, 0=q_{0}<q_{1}<\cdots<q_{N}=P_{M}$. Let $\phi_{k}$ be the piecewise linear spline defined by $\phi_{k}\left(q_{l}\right)=\delta_{k l}$ where $\delta_{k l}$ is the Kronecker delta. Let us consider now the $J$-interval, $I=\left[0, P_{M}\right] \times \cdots \times\left[0, P_{M}\right] \subset \mathbb{R}^{J}$. We consider $\mathbb{S}_{1}^{0}$ the space of $J$-linear splines in $I$ defined by

$$
s\left(p_{1}, \ldots, p_{J}\right)=\sum_{\nu_{1}, \ldots, \nu_{J}=0}^{N} \hat{s}_{\nu_{1}, \ldots, \nu_{J}} \phi_{\nu_{1}}\left(p_{1}\right) \ldots \phi_{\nu_{J}}\left(p_{J}\right) .
$$

Note that by construction the coefficients $\hat{s}_{\nu_{1}, \ldots, \nu_{J}}$ are determined by $\hat{s}_{\nu_{1}, \ldots, \nu_{J}}=s\left(q_{\nu_{1}}, \ldots, q_{\nu_{J}}\right)$. We introduce the notation $\boldsymbol{q}_{\nu_{1}, \ldots, \nu_{J}}=\left[q_{\nu_{1}}, \ldots, q_{\nu_{J}}\right]^{J}, \nu_{i}=0, \ldots, N, i=1, \ldots, J$.

The approximation $V_{h, i}^{N} \in \mathbb{S}_{1}^{0}$ to the time-discrete value function $V_{h, i}$ in (69) is computed by a fixed-point iteration solving for $r \geq 0$, and $i=1, \ldots, J$,

$$
V_{h, i}^{N,[r+1]}\left(\boldsymbol{q}_{\nu_{1}, \ldots, \nu_{J}}\right)=\max _{u_{i} \geq 0}\left\{h \widetilde{G}_{i}\left(q_{\nu_{i}}, u_{i}\right)+\delta V_{h, i}^{N,[r]}\left(\boldsymbol{q}_{\nu_{1}, \ldots, \nu_{J}}+h \boldsymbol{g}\left(\boldsymbol{q}_{\nu_{1}, \ldots, \nu_{J}},\left[u_{i}, \boldsymbol{u}_{-i, \nu_{1}, \ldots, \nu_{J}}^{[r]}\right]\right)\right)\right\},
$$

where

$$
u_{i, \nu_{1}, \ldots, \nu_{J}}^{[r+1]}=\arg \max _{u_{i} \geq 0}\left\{h \widetilde{G}_{i}\left(q_{\nu_{i}}, u_{i}\right)+\delta V_{h, i}^{N,[r]}\left(\boldsymbol{q}_{\nu_{1}, \ldots, \nu_{J}}+h \boldsymbol{g}\left(\boldsymbol{q}_{\nu_{1}, \ldots, \nu_{J}},\left[u_{i}, \boldsymbol{u}_{-i, \nu_{1}, \ldots, \nu_{J}}^{[r]}\right]\right)\right)\right\} .
$$


The iteration is initialized with some given $V_{h, i}^{N,[0]}\left(\boldsymbol{q}_{\nu_{1}, \ldots, \nu_{J}}\right)$ and $u_{i, \nu_{1}, \ldots, \nu_{J}}^{[0]}$, for $\nu_{i}=0, \ldots, N, i=$ $1, \ldots, J$ and stopped when

$$
\left|V_{h, i}^{N,[r+1]}\left(\boldsymbol{q}_{\nu_{1}, \ldots, \nu_{J}}\right)-V_{h, i}^{N,[r]}\left(\boldsymbol{q}_{\nu_{1}, \ldots, \nu_{J}}\right)\right|<\mathrm{TOL}
$$

for all possible values of the indices $\nu_{1}, \ldots, \nu_{J}$ and $i$. The parameter TOL is a prescribed positive tolerance.

When the iteration is stopped the approximated time-discrete value function is defined as the last

iterant $V_{h, i}^{N}:=V_{h, i}^{N,[r+1]}$. The approximated time-discrete optimal policies are defined as the unique $J$-linear spline $u_{h, N, i}^{*}$ interpolating the values $u_{i, \nu_{1}, \ldots, \nu_{J}}^{[r+1]}, \nu_{i}=0, \ldots, N, i=1, \ldots, J$. The approximated time-discrete optimal state trajectory is computed from

$$
\boldsymbol{p}_{n+1}^{*}=\boldsymbol{p}_{n}^{*}+h \boldsymbol{g}\left(\boldsymbol{p}_{n}^{*}, \boldsymbol{u}_{n}^{*}\right), \quad n \geq 0,
$$

where $\boldsymbol{u}_{n}^{*}=\left[u_{h, M, 1}^{*}\left(\boldsymbol{p}_{n}^{*}\right), \ldots, u_{h, M, J}^{*}\left(\boldsymbol{p}_{n}^{*}\right)\right]^{T}$ and $\boldsymbol{p}_{0}^{*}=\boldsymbol{p}_{0}$.

\section{References}

[1] Anita, S., Capasso, V., Kunze, H., La Torre, D.: Optimal Control and Long-Run Dynamics for a Spatial Economic Growth Model with Physical Capital Accumulation and Pollution Diffusion. Applied Mathematics Letters 26, 908-912 (2013).

[2] Barbu, V.: Analysis and Control of Nonlinear Infinite Dimensional Systems, Academic Press, Boston. 1993.

[3] Başar, T., Olsder, G.J.: Dynamic Noncooperative Game Theory, SIAM, Philadelpia, 1999.

[4] Benchekroun, H., Long, N.V.: Efficiency Inducing Taxation for Polluting Oligopolists. Journal of Public Economics 70, 325-342 (1998).

[5] Brito, P.: The Dynamics of Growth and Distribution in a Spatially Heterogenous World. WP13/2004/DE/UECE, Technical University of Lisbon (2004).

[6] Brock, W., Xepapadeas, A.: General Pattern Formation in Recursive Dynamic Systems Models in Economics. MPRA PaperNo. 12305, University of Munich (2008).

[7] Brock, W., Xepapadeas, A.: Diffusion-Induced Instability and Pattern Formation in Infinite Horizon Recursive Optimal Control. Journal of Economic Dynamics \& Control 32, 2745-2787 (2008).

[8] Brock, W., Xepapadeas, A.: Pattern Formation, Spatial Externalities and Regulation in Coupled Economic-Ecological Systems. Journal of Environmental Economics and Management 59, 149-164 (2010). 
[9] Brock, W., Xepapadeas, A., Yannacopoulos, A.N.: Optimal Agglomerations in Dynamic Economics. Journal of Mathematical Economics 53, 1-15 (2014).

[10] Brock, W., Xepapadeas, A., Yannacopoulos, A.N.: Optimal Control in Space and Time and the Management of Environmental Resources. Annual Reviews of Resource Economics 6, 33-68 (2014).

[11] Boucekkine, R., Camacho, C., Zou, B.: Bridging the Gap Between Growth Theory and the New Economic Geography: The Spatial Ramsey Model. Macroeconomic Dynamics 13, 20-45 (2009).

[12] Boucekkine, R., Camacho, C., Fabbri, G.: Spatial Dynamics and Convergence: The Spatial Ak Model. Journal of Economic Theory 148, 2719-2736 (2013).

[13] Boucekkine, R., Camacho, C., Fabbri, G.: On the Optimal Control of Some Parabolic Partial Differential Equations Arising in Economics. Serdica Mathematical Journal 39, 331-354 (2013).

[14] Camacho, C., Pérez-Barahona, A.: Land Use Dynamics and the Environment. Journal of Economic Dynamics \& Control 52, 96-118 (2015).

[15] Camacho, C., Zou, B., Briani, M.: On the Dynamics of Capital Accumulation Across Space. European Journal of Operational Research 186, 451-465 (2008).

[16] Cannarsa, P., Da Prato, G.: Some Results on Nonlinear Optimal Control Problems and HamiltonJacobi Equations in Infinite Dimensions. Journal of Functional Analysis 90, 27-47 (1990).

[17] De Frutos, J., Martín-Herrán, G.: Does Flexibility Facilitate Sustainability of Cooperation Over Time? A Case Study from Environmental Economics. Journal of Optimization Theory and Applications 165(2), 657-677 (2015).

[18] Desmet, K., Rossi-Hansberg, E.: On Spatial Dynamics. Journal of Regional Science 50, 43-63 (2010).

[19] Desmet, K., Rossi-Hansberg, E.: On Spatial Economic Impact of Global Warming. Journal of Urban Economics 88, 16-37 (2015).

[20] Dockner, E.J., Long, N.V.: International Pollution Control: Cooperative versus Noncooperative Strategies. Journal of Environmental Economics and Management 25, 13-29 (1993).

[21] Fabbri, G.: Ecological Barriers and Convergence: A Note on Geometry in Spatial Growth Models. Journal of Economic Theory 162, 114-136 (2016).

[22] Graß, D., Uecker, H.: Optimal Management and Spatial Patterns in a Distributed Shalow Lake Model. Electronic Journal of Differential Equations 2017(1), 1-21 (2017).

[23] Hager, W. W.: Runge-Kutta Methods in Optimal Control and the Transformed Adjoint System. Numerische Mathematik 87, 247-282 (2000). 
[24] Haurie, H., Krawczyk, J.B., Zaccour, G.: Games and Dynamic Games, World Scientific, Singapore, 2012.

[25] Jørgensen, S., Martín-Herrán, G., Zaccour, G.: Dynamic Games in the Economics and Management of Pollution. Environmental Modeling and Assessment 15, 433-467 (2010).

[26] Li, X., Yong, J.: Optimal Control Theory for Infinite Dimensional Systems, Birkhäuser, Boston, 1995.

[27] La Torre, D., Liuzzi, D., Marsiglio, S.: Pollution Diffusion and Abatement Activities across Space and over Time. Mathematical Social Sciences 78, 48-63 (2015).

[28] Mäler, K-G., De Zeeuw, A.: The Acid Rain Differential Game. Environmental and Resource Economics 12, 167-184 (1998).

[29] Mäler, K-G., Xepapadeas, A., De Zeeuw, A.: The Economics of Shallow Lakes. Environmental and Resource Economics 26, 603-624 (2003).

[30] Transboundary Waters: Sharing Benefits, Sharing Responsibilities, UN-Water Thematic paper, www.unwater.org/downloads/UNW_TRANSBOUNDARY.pdf, 2008, downloaded April 10, 2017.

[31] Stockie, J.M.: The Mathematics of Atmospheric Dispersion Modeling. SIAM Review 53, 349-372 (2011).

[32] Tröltzsch, F.: Optimal Control of Partial Differential Equations. Theory, Methods and Applications, AMS, Providence, 2009.

[33] Van der Ploeg, F., De Zeeuw, A.J.: International Aspects of Pollution Control. Environmental and Resource Economics 2, 117-139 (1992).

[34] Xepapadeas, A.: Environmental Policy Design and Dynamic Nonpoint-Source Pollution. Journal of Environmental Economics and Management 23, 22-39 (1992).

[35] Xepapadeas, A.: The Spatial Dimension in Environmental and Resource Economics. Environment and Development Economics 15, 747-758 (2010).

[36] Yamaguchi, R.: Sustainability and Spatial Externality in a Capital-Resource Economy. Mimeo (2014). 